\title{
Constant-to-one and onto global maps of homomorphisms between strongly connected graphs
}

\author{
MASAKAZU NASU \\ Faculty of Engineering, Mie University, Tsu 514, Japan \\ (Received 25 August 1982 and revised 28 March 1983)
}

\begin{abstract}
The global maps of homomorphisms of directed graphs are very closely related to homomorphisms of a class of symbolic dynamical systems called subshifts of finite type. In this paper, we introduce the concepts of 'induced regular homomorphism' and 'induced backward regular homomorphism' which are associated with every homomorphism between strongly connected graphs whose global map is finite-to-one and onto, and using them we study the structure of constant-toone and onto global maps of homorphisms between strongly connected graphs and that of constant-to-one and onto homomorphisms of irreducible subshifts of finite type. We determine constructively, up to topological conjugacy, the subshifts of finite type which are constant-to-one extensions of a given irreducible subshift of finite type. We give an invariant for constant-to-one and onto homomorphisms of irreducible subshifts of finite type.
\end{abstract}

\section{Introduction}

A homomorphism between graphs (the word 'graph' means 'directed graph' throughout this paper), naturally induces a mapping between the bisequence spaces over the graphs, which is called the global map of the homomorphism. The bisequence spaces $\Omega(G)$ over graphs $G$ with the shift homeomorphisms $\sigma$ on them constitute a class of symbolic dynamical systems $(\Omega(G), \sigma)$ called subshifts of finite type (or topological Markov chains) and hence the global map of a homomorphism of graphs is a homomorphism of subshifts of finite type. The converse of this is almost valid by the theorem of Curtis, Hedlund and Lyndon [8]. Therefore, many properties of the global maps of homomorphisms of graphs can straightforwardly be interpreted as those of homomorphisms of subshifts of finite type, so that the study of the global maps of homomorphisms of graphs provides useful combinatorial approaches to that of homomorphisms of subshifts of finite type (cf. [17]). In fact, in [1] and others, a notion similar to that of a homomorphism of graphs was used as a one-block map together with other graph theoretical notions for the study of homomorphisms of subshifts of finite type.

On the other hand, the global maps of homomorphisms of graphs and homomorphisms of subshifts of finite type can be considered as a new area of graph theory which investigates relations between graphs, especially in connection with the 
spectral properties of the adjacency matrices of graphs. In fact, [17] treated this, and moreover the results in the classification theories for subshifts of finite type of [21], [18], and [1] and the results on homomorphisms of subshifts of finite type of [13] and [10] can be interpreted as results in graph theory concerning the above area.

In this paper, we introduce the concepts of 'induced regular homomorphism' and 'induced backward regular homomorphism' which are associated with every homomorphism between strongly connected graphs whose global map is finite-toone and onto. Using them we study constant-to-one and onto global maps of homomorphisms between strongly connected graphs and constant-to-one and onto homomorphisms of irreducible subshifts of finite type. (The term 'constant-to-one' means ' $k$-to-one for some $k$ '.) We give some necessary and sufficient conditions for the global map of a homomorphism between strongly connected graphs to be constant-to-one and onto, one of which immediately gives a structure result for constant-to-one and onto homomorphisms of irreducible subshifts of finite type (corollary 6.4). Using this we obtain our main theorem (theorem 7.3) which determines constructively, up to topological conjugacy, the subshifts of finite type which are constant-to-one extensions of a given irreducible subshift of finite type (that is, the subshifts of finite type such that there are constant-to-one and onto homomorphisms from them to a given irreducible subshift of finite type). It is also shown that if there exists a constant-to-one and onto homomorphism from a subshift of finite type $\left(\Omega\left(G_{1}\right), \sigma_{1}\right)$ to an irreducible subshift of finite type $\left(\Omega\left(G_{2}\right), \sigma_{2}\right)$, then the elementary divisors not divisible by $\lambda$ (the indeterminate) of the adjacency matrix of $G_{2}$ are contained in the elementary divisors of the adjacency matrix of $G_{1}$.

Many extended notions, techniques and results of those in [8] appear in this paper; the reader is assumed to be familiar with [8].

Many statements of the theorems, propositions, and lemmas after $\S 2$ contain second versions. But proofs will be given only for the first versions because the proofs of the second versions are similar.

This work was done when the author was at the Research Institute of Electrical Communication, Tohoku University.

\section{Background}

A graph (directed graph with labelled points and labelled arcs) $G$ is defined to be a triple $(P, A, \zeta)$ where $P$ is a finite set of elements called points, $A$ is a finite set of elements called arcs and $\zeta$ is a mapping of $A$ into $P \times P$. If $\zeta(a)=(u, v)$ for $a \in A$ and $u, v \in P$, then $u$ and $v$ are the initial endpoint of $a$ and the terminal endpoint of $a$, respectively, which are denoted by $\mathrm{i}(a)$ and $\mathrm{t}(a)$, respectively.

A sequence $x=a_{1} \cdots a_{p}(p \geq 1)$ with $a_{i} \in A, i=1, \ldots, p$, is a path of length $p$ in $G$ if

$$
\mathrm{t}\left(a_{i}\right)=\mathrm{i}\left(a_{i+1}\right) \quad \text { for } i=1, \ldots, p-1 .
$$

We call $\mathrm{i}\left(a_{1}\right)$ and $\mathrm{t}\left(a_{p}\right)$ the initial endpoint of $x$ and the terminal endpoint of $x$, respectively. Every point $u$ of $G$ is a path of length 0 in $G$ whose initial and terminal endpoint is $u$. For any path $x$ in $G$, we denote by $\mathrm{i}(x)$ and $\mathrm{t}(x)$ the initial endpoint of $x$ and the terminal endpoint of $x$, respectively, and if $\mathrm{i}(x)=u$ and $\mathrm{t}(x)=v$, then 
we often say that $x$ goes from $u$ to $v$. The set of all paths in $G$ is denoted by $\Pi(G)$. The set of all paths of length $p \geq 0$ in $G$ is denoted by $\Pi^{(p)}(G)$.

Standing hypothesis. Throughout the remainder of this paper, we assume that a graph has at least one point and for each point $u$, there exists at least one arc going to $u$ and at least one arc going from $u$.

Let $\mathbb{Z}$ be the set of integers. Let $G=(P, A, \zeta)$ be a graph. A mapping $\alpha: \mathbb{Z} \rightarrow A$ is a bisequence over $G$ if

$$
\mathrm{t}(\alpha(i))=\mathrm{i}(\alpha(i+1)) \quad \text { for all } i \in \mathbb{Z} .
$$

Let $\Omega(G)$ denote the set of all bisequences over $G$. If $\alpha \in \Omega(G)$ and $i \in \mathbb{Z}$, then $\alpha(i)$ will often be denoted by $\alpha_{i}$. For $\alpha \in \Omega(G)$ and $i, j \in \mathbb{Z}$ with $i \leq j$, let

$$
\alpha[i, j]=\alpha_{i} \alpha_{i+1} \cdots \alpha_{j}
$$

Clearly $\alpha[i, j] \in \Pi^{(j-i+1)}(G)$. We define a metric $d$ on $\Omega(G)$ as follows: let $\alpha, \beta \in$ $\Omega(G)$,

$$
\begin{aligned}
& d(\alpha, \beta)=0 \quad \text { if } \alpha=\beta, \\
& d(\alpha, \beta)=(1+k)^{-1} \quad \text { if } \alpha \neq \beta
\end{aligned}
$$

where

$$
k=\min \{i \geq 0 \mid \alpha[-i, i] \neq \beta[-i, i]\} .
$$

With this metric, $\Omega(G)$ is compact.

Let $G_{1}=\left(P, A, \zeta_{1}\right)$ and $G_{2}=\left(Q, B, \zeta_{2}\right)$ be graphs. A homomorphism of $G_{1}$ into $G_{2}$ is a pair $(h, \phi)$ of mappings $h: A \rightarrow B$ and $\phi: P \rightarrow Q$ such that for any $a \in A$, if $\zeta_{1}(a)=(u, v)$ with $u, v \in P$, then

$$
\zeta_{2}(h(a))=(\phi(u), \phi(v)) .
$$

By our standing hypothesis for graphs, the homomorphisms $(h, \phi)$ of $G_{1}$ into $G_{2}$ is uniquely determined by $h$. Therefore, we say that $h$ is a homomorphism of $G_{1}$ into $G_{2}$ and we denote by $\phi_{h}$ the unique mapping $\phi$ such that $(h, \phi)$ is a homomorphism of $G_{1}$ into $G_{2}$.

A homomorphism $h: A \rightarrow B$ of a graph $G_{1}=\left(P, A, \zeta_{1}\right)$ into a graph $G_{2}=\left(Q, B, \zeta_{2}\right)$ is naturally extended to a mapping

$$
h^{*}: \Pi\left(G_{1}\right) \rightarrow \Pi\left(G_{2}\right) \text {. }
$$

That is, we define $h^{*}: \Pi\left(G_{1}\right) \rightarrow \Pi\left(G_{2}\right)$ as follows: for each $x \in \Pi\left(G_{1}\right)$, if the length of $x$ is 0 , i.e. $x$ is a point of $G_{1}$, then $h^{*}(x)=\phi_{h}(x)$, and if $x=a_{1} \cdots a_{p}(p \geq 1)$ with $a_{i} \in A, i=1, \ldots, p$, then $h^{*}(x)=h\left(a_{1}\right) \cdots h\left(a_{p}\right)$. The mapping $h^{*}$ is called the extension of $h$. Another mapping is naturally induced by $h$. We define

$$
h_{\infty}: \Omega\left(G_{1}\right) \rightarrow \Omega\left(G_{2}\right)
$$

as follows: for $\alpha \in \Omega\left(G_{1}\right), h_{\infty}(\alpha)=\beta$, where $\beta_{i}=h\left(\alpha_{i}\right)$ for all $i \in \mathbb{Z}$. We call $h_{\infty}$ the global map of $h$. A graph $G=(P, A, \zeta)$ is strongly connected if for any $u, v \in P$, there exists a path going from $u$ to $v$.

For a positive integer $k$, a mapping $f: X \rightarrow Y$ is $k$-to-one if $\left|f^{-1}(y)\right|=k$ for all $y \in f(X)$. A mapping $f: X \rightarrow Y$ is constant-to-one if there exists a positive integer $k$ such that $f$ is k-to-one; uniformly finite-to-one if there exists a positive integer $k$ such that $\left|f^{-1}(y)\right| \leq k$ for all $y \in Y$; and finite-to-one if $\left|f^{-1}(y)\right|<\infty$ for all $y \in Y$. 
Let $h$ be a homomorphism of a graph $G_{1}$ into a graph $G_{2}$. Two paths $x$ and $y$ in $G_{1}$ are indistinguishable by $h$ if

$$
\mathrm{i}(x)=\mathrm{i}(y), \quad \mathrm{t}(x)=\mathrm{t}(y), \quad \text { and } \quad h^{*}(x)=h^{*}(y) .
$$

The following results are found in [17]. Similar results also appear in [1].

Proposition 1.1. Let $h$ be a homomorphism of a strongly connected graph $G_{1}$ into a graph $G_{2}$. Then the following statements are equivalent.

(1) No two distinct paths in $G_{1}$ are indistinguishable by $h$.

(2) $h^{*}$ is uniformly finite-to-one.

(3) $h_{\infty}$ is uniformly finite-to-one.

(4) $h_{\infty}$ is finite-to-one.

Proposition 1.2. For any homomorphism $h$ of a graph $G_{1}$ into a graph $G_{2}, h^{*}$ is onto iff $h_{\infty}$ is onto.

For a graph $G$, we denote by $M(G)$ the adjacency matrix of $G$ (i.e. if $G$ has $n$ points $u_{1}, \ldots, u_{n}$, then $M(G)$ is the square matrix $\left(m_{i j}\right)$ of order $n$ such that $m_{i j}$ is the number of arcs going from $u_{i}$ to $u_{j}$ ). Since $M(G)$ is a non-negative matrix, by the Perron-Frobenius Theorem, $M(G)$ has the non-negative characteristic value that the moduli of all the other characteristic values do not exceed (cf. [6, Vol. II]). We denote by $r(G)$ that 'maximal' characteristic value of $M(G)$.

The following result is found in [17]. In view of the above propositions and the facts stated later in this section, it is essentially the same as the well-known result on symbolic flows (see, e.g., [1]) that a finite-to-one and onto homomorphism (of symbolic flows) between subshifts of finite type preserves topological entropy.

Proposition 1.3. If there is a homomorphism $h$ of a graph $G_{1}$ into a graph $G_{2}$ with $h^{*}$ uniformly finite-to-one and onto, then $r\left(G_{1}\right)=r\left(G_{2}\right)$.

In [17], a stronger result has been given. That is, it has been proved there that with the same condition as in proposition 1.3, not only $r\left(G_{1}\right)=r\left(G_{2}\right)$ but also the characteristic polynomial of $M\left(G_{2}\right)$ divides that of $M\left(G_{1}\right)$. Furthermore, Kitchens ([10]) has given a still stronger result. He has proved that if $G_{1}$ and $G_{2}$ are strongly connected graphs and $M\left(G_{1}\right)$ and $M\left(G_{2}\right)$ are $0-1$ matrices and if there is a homomorphism $h$ of $G_{1}$ into $G_{2}$ with $h_{\infty}$ finite-to-one and onto, then the block of the Jordan form of $M\left(G_{2}\right)$ is a principal submatrix of the Jordan form of $M\left(G_{1}\right)$. The condition that $M\left(G_{1}\right)$ and $M\left(G_{2}\right)$ are $0-1$ matrices can be eliminated.

The following result has been proved in [17] using a graph-theoretical method. In view of propositions 1.1 and 1.2 , it can also follow from a result in [4].

THEOREM 1.4. Let $G_{1}$ and $G_{2}$ be two strongly connected graphs with $r\left(G_{1}\right)=r\left(G_{2}\right)$. Then for any homomorphism $h$ of $G_{1}$ into $G_{2}, h^{*}$ is uniformly finite-to-one iff $h^{*}$ is onto.

Let $G_{1}$ and $G_{2}$ be strongly connected graphs and let $h$ be a homomorphism of $G_{1}$ into $G_{2}$. Then, by the above propositions and theorem 1.4 , we have many equivalent 
statements; the following are several of them.

(1) $r\left(G_{1}\right)=r\left(G_{2}\right)$ and $h^{*}$ is onto.

(2) $h^{*}$ is uniformly finite-to-one and onto.

(3) $h_{\infty}$ is finite-to-one and onto.

(4) $h^{*}$ is onto and there exist no two distinct paths which are indistinguishable by $h$.

(5) $r\left(G_{1}\right)=r\left(G_{2}\right)$ and there exist no two distinct paths which are indistinguishable by $h$.

We will use (1) as a representative of these and the other equivalent statements in most statements of conditions in our results. Moreover the equivalence of them will be used frequently without reference.

Let $G=(P, A, \zeta)$ be a graph. For any non-negative integer $p$, we define a graph $L^{(p)}(G)$ as follows: $L^{(0)}(G)=G$. For $p \geq 1, L^{(p)}(G)=\left(\Pi^{(p)}(G), \Pi^{(p+1)}(G), \zeta^{(p)}\right)$, where $\zeta^{(p)}\left(a_{1} \cdots a_{p+1}\right)=\left(a_{1} \cdots a_{p}, a_{2} \cdots a_{p+1}\right)$ for $a_{1} \cdots a_{p+1} \in \Pi^{(p+1)}(G)$ with $a_{i} \in A, i=1, \ldots, p+1$. We call $L^{(p)}(G)$ the path graph of length $p$ of $G$. $\left(L^{(1)}(G)\right.$ is usually known as the line digraph of $G$ (cf. [7]) or the adjoint of $G$ (cf. [2]).) Essentially the same notion as $L^{(p)}(G)$ was also used for 'higher block system' of [1].) Clearly, if $G$ is strongly connected, then $L^{(p)}(G)$ is strongly connected for all $p \geq 0$. For any integers $p$ and $q$ with $p \geq q \geq 1$, we define a mapping

$$
h_{G, p, q}: \Pi^{(p)}(G) \rightarrow A
$$

as follows. For any $a_{1} \cdots a_{p} \in \Pi^{(p)}(G)$ with $a_{i} \in A$,

$$
h_{G, p, q}\left(a_{1} \cdots a_{p}\right)=a_{q} .
$$

Then clearly $h_{G, p, q}$ is a homomorphism of $L^{(p-1)}(G)$ into $G$ and $\left(h_{G, p, q}\right)^{*}$ is uniformly finite-to-one and onto. Hence by proposition 1.3,

$$
r\left(L^{(p-1)}(G)\right)=r(G) .
$$

Furthermore $\left(h_{G, p, q}\right)_{\infty}$ is a homeomorphism of $\Omega\left(L^{(p-1)}(G)\right)$ onto $\Omega(G)$.

Let $A$ be a finite non-empty set (of symbols). Let $G_{0}(A)$ be the graph defined by

$$
G_{0}(A)=\left(\{\Lambda\}, A, \zeta_{A}\right)
$$

where $\zeta_{A}(a)=(\Lambda, \Lambda)$ for all $a \in A$. Then $G_{0}(A)$ is a strongly connected graph having only one point $\Lambda$ and each element of $A$ is an arc (loop) going from $\Lambda$ to itself. Clearly $\Pi\left(G_{0}(A)\right)$ is the set of all finite sequences of elements of $A$ and

$$
\Omega\left(G_{0}(A)\right)=A^{\mathbb{Z}} \text {. }
$$

Let $\Omega_{A}=A^{\mathbb{Z}}$. Each element of $\Omega_{A}$ is a bisequence over $A$. Of course, $\Omega_{A}$ is a compact metric space with the metric defined before. The homeomorphism $\sigma: \Omega_{A} \rightarrow$ $\Omega_{A}$ defined by

$$
(\sigma(\alpha))_{i}=\alpha_{i+1}, \quad \alpha \in \Omega_{A}, \quad i \in \mathbb{Z},
$$

is called the shift. The dynamical system $\left(\Omega_{A}, \sigma\right)$ is called the full shift system over $A$. Let $X$ be a closed non-empty subset of $\Omega_{A}$ such that $\sigma^{-1}(X)=X$. The dynamical system $(X, \sigma)$ is called a subdynamical system of $\left(\Omega_{A}, \sigma\right)$ or a symbolic flow over $A$. (For simplicity, we denote $\sigma \mid X$ by $\sigma$.) 
Let $G=(P, A, \zeta)$ be a graph. Then $(\Omega(G), \sigma)$ is a subdynamical system of $\left(\Omega_{A}, \sigma\right)$. A symbolic flow of this type is called a subshift of finite type, (cf. [21] and [19]). If $G$ is strongly connected, then $(\Omega(G), \sigma)$ is an irreducible subshift of finite type. (Cf. [3]. Note that $G$ is strongly connected iff the adjacency matrix $M(G)$ of $G$ is irreducible.)

Let $\left(X, \sigma_{1}\right)$ and $\left(Y, \sigma_{2}\right)$ be two symbolic flows. A homomorphism $\pi:\left(X, \sigma_{1}\right) \rightarrow$ $\left(Y, \sigma_{2}\right)$ is a continuous mapping of $X$ into $Y$ such that $\pi \sigma_{1}=\sigma_{2} \pi$. We say that $\left(X, \sigma_{1}\right)$ and $\left(Y, \sigma_{2}\right)$ are topologically conjugate if there exists an isomorphism of $\left(X, \sigma_{1}\right)$ onto $\left(Y, \sigma_{2}\right)$.

Clearly global maps of homomorphisms of graphs are homomorphisms of symbolic flows. The converse is almost valid.

Let $G_{1}=\left(P, A, \zeta_{1}\right)$ and $G_{2}=\left(Q, B, \zeta_{2}\right)$ be two graphs and let $p$ be a positive integer. A mapping $f: \Pi^{(p)}\left(G_{1}\right) \rightarrow B$ is an admissible p-block map if for any $a_{1} \cdots a_{p+1} \in \Pi^{(p+1)}\left(G_{1}\right)$ with $a_{1}, \ldots, a_{p+1} \in A$,

$$
\mathrm{t}\left(f\left(a_{1} \cdots a_{p}\right)\right)=\mathrm{i}\left(f\left(a_{2} \cdots a_{p+1}\right)\right) .
$$

Corresponding to any admissible $p$-block map $f: \Pi^{(p)}\left(G_{1}\right) \rightarrow B$, we define a mapping $f_{\infty}: \Omega\left(G_{1}\right) \rightarrow \Omega\left(G_{2}\right)$ by

$$
f_{\infty}(\alpha)=\beta \quad \text { where } \beta_{i}=f\left(\alpha_{i} \cdots \alpha_{i+p-1}\right)
$$

for all $i \in \mathbb{Z}$. Clearly an admissible 1-block map $f$ is a homomorphism of $G_{1}$ into $G_{2}$ and $f_{\infty}$ is its global map. The well-known theorem of Curtis, Hedlund \& Lyndon [8] for homomorphisms of symbolic flows (as pointed out by Klein [12]) implies the following.

THeorem 1.5. (Curtis, Hedlund \& Lyndon) Let $G_{1}$ and $G_{2}$ be graphs. Then a mapping $\pi: \Omega\left(G_{1}\right) \rightarrow \Omega\left(G_{2}\right)$ is a homomorphism of $\left(\Omega\left(G_{1}\right), \sigma_{1}\right)$ into $\left(\Omega\left(G_{2}\right), \rho_{2}\right)$ iff there exist integers $p \geq 1$ and $k$ and an admissible p-block map

$$
f: \Pi^{(p)}\left(G_{1}\right) \rightarrow \Pi^{(1)}\left(G_{2}\right)
$$

such that

$$
\pi=\sigma^{-k} f_{\infty}
$$

The following, which appears in [17], is a graph-theoretical interpretation of the above theorem.

COROLlary 1.6. Let $G_{1}$ and $G_{2}$ be graphs. Then a mapping $\pi: \Omega\left(G_{1}\right) \rightarrow \Omega\left(G_{2}\right)$ is a homomorphism of $\left(\Omega\left(G_{1}\right), \sigma_{1}\right)$ into $\left(\Omega\left(G_{2}\right), \sigma_{2}\right)$ iff there exist integers $p$ and $q$ with $p \geq q \geq 1$ and a homomorphism $h$ of $L^{(p-1)}\left(G_{1}\right)$ into $G_{2}$ such that

$$
\pi=h_{\infty}\left(h_{G_{1}, p, q}\right)_{\infty}^{-1} \text {. }
$$

We remark that for a graph $G$ and integers $p$ and $q$ with $p \geq q \geq 1,\left(h_{G, p, q}\right)_{\infty}$ is an isomorphism of $\left(\Omega\left(L^{(p-1)}(G)\right), \sigma^{\prime}\right)$ onto $(\Omega(G), \sigma)$.

\section{Regular homomorphisms and biregular homomorphisms}

A homomorphism $h$ of a graph $G_{1}$ into a graph $G_{2}$ is regular [backward-regular (abbreviated to $b$-regular)] if for each point $u$ of $G_{1}$ and for each arc $b$ going from 
[to] $\phi_{h}(u)$, there exists exactly one arc $a$ going from [to] $u$ with $h(a)=b$. The same notions appear in [1] as 'right-resolving' ['left-resolving'] together with the following.

Proposition 2.1. If $h$ is a regular [b-regular] homomorphism of a graph $G_{1}$ into a strongly connected graph $G_{2}$, then $h^{*}$ and $h_{\infty}$ are uniformly finite-to-one and onto and $r\left(G_{1}\right)=r\left(G_{2}\right)$.

A homomorphism $h$ is biregular if $h$ is both regular and b-regular.

Proposition 2.2. Let $h$ be a biregular homomorphism of a graph $G_{1}=\left(P, A, \zeta_{1}\right)$ into a strongly connected graph $G_{2}=\left(Q, B, \zeta_{2}\right)$. Then $h^{*}$ and $h_{\infty}$ are $|P| /|Q|$-to-one and onto.

Proof. By proposition 2.1, $h^{*}$ and $h_{\infty}$ are onto. Since $h$ is regular, it follows that

$$
\left|\left(h^{*}\right)^{-1}(y)\right|=\left|\phi_{h}^{-1}(\mathrm{i}(y))\right| \quad \text { for all } y \in \Pi\left(G_{2}\right) \text {. }
$$

Since $h$ is b-regular, it also follows that

$$
\left|\left(h^{*}\right)^{-1}(y)\right|=\left|\phi_{h}^{-1}(\mathbf{t}(y))\right| \quad \text { for all } y \in \Pi\left(G_{2}\right) .
$$

Let $v_{1}, v_{2} \in Q$. Since $G_{2}$ is strongly connected, there exists a path $y$ in $G_{2}$ going from $v_{1}$ to $v_{2}$. Hence, by the above,

$$
\left|\phi_{h}^{-1}\left(v_{1}\right)\right|=\left|\left(h^{*}\right)^{-1}(y)\right|=\left|\phi_{h}^{-1}\left(v_{2}\right)\right| .
$$

Thus, for all $v \in Q,\left|\phi_{h}^{-1}(v)\right|=|P| /|Q|$, and hence for all $y \in \Pi\left(G_{2}\right)$,

$$
\left|\left(h^{*}\right)^{-1}(y)\right|=|P| /|Q| \text {. }
$$

Thus $h^{*}$ is $|P| /|Q|$-to-one.

For each $\beta \in \Omega\left(G_{2}\right)$,

$$
\left|h_{\infty}^{-1}(\beta)\right| \leq\left(h^{*}\right)^{-1}(\beta[-i, i])
$$

for some sufficiently large integer $i \geq 0$. Hence $\left|h_{\infty}^{-1}(\beta)\right| \leq|P| /|Q|$. Thus it suffices to show that for each $\beta \in \Omega\left(G_{2}\right)$, there exist at least $|P| /|Q|$ bisequences $\alpha$ such that $h_{\infty}(\alpha)=\beta$. This is proved in a similar way to that used in the proof of theorem 6.7 of Hedlund [8].

For a graph $G$, we call a graph $G_{1}$ such that there exists a biregular homomorphism $h$ of $G_{1}$ into $G$ a biregular extension of $G$. Given a strongly connected graph $G$, it is easy to determine all biregular extensions $G_{1}$ and biregular homomorphisms $h$ of $G_{1}$ into $G$.

Let $h$ be a biregular homomorphism of a graph $G_{1}=\left(P, A, \zeta_{1}\right)$ into a strongly connected graph $G_{2}=\left(Q, B, \zeta_{2}\right)$. Since $h$ is regular, for each $b \in B$, we can define a mapping

$$
\mu_{b}: \phi_{h}^{-1}(\mathrm{i}(b)) \rightarrow \phi_{h}^{-1}(\mathrm{t}(b))
$$

as follows. For each $u \in \phi_{h}^{-1}(\mathrm{i}(b))$, define $\mu_{b}(u)=\mathrm{t}(a)$, where $a$ is the unique arc of $G_{1}$ such that $\mathrm{i}(a)=u$ and $h(a)=b$. Since $h$ is b-regular, it follows that $\mu_{b}$ is a bijection. Since $G_{2}$ is strongly connected, by proposition 2.2 there exists a positive integer $k$ such that $\left|\phi_{h}^{-1}(v)\right|=k$ for all $v \in Q$. Thus for each $b \in B, \mu_{b}$ is a bijection of a $k$-point set onto a $k$-point set. 
Let the adjacency matrix $M\left(G_{2}\right)$ of $G_{2}$ be $\left(m_{i j}\right)$. Then it is easy to see that the adjacency matrix of $G_{1}$ can be written in the form

$$
M\left(G_{1}\right)=\left(\begin{array}{lll}
M_{11} & \cdots & M_{1 q} \\
& \cdots & \\
M_{q 1} & \cdots & M_{q q}
\end{array}\right)
$$

where $q=|Q|$ and $M_{i j}$ is the square matrix of order $k$ obtained by summing the $m_{i j}$ permutation matrices corresponding to the $\mu_{b}$ 's such that $b$ 's are the arcs of $G_{2}$ going from point $i \in Q$ to point $j \in Q$. (If $m_{i j}=0$, then $M_{i j}$ is the zero-matrix of order $k$.)

Conversely, if we are given an assignment of some permutation $\mu_{b}$ on $\{1, \ldots, k\}$ to each arc $b \in B$ of a strongly connected graph $G_{2}=\left(Q, B, \zeta_{2}\right)$, then we can straightforwardly obtain a biregular extension of $G_{2}$ and a biregular homomorphism $h$ of $G_{1}$ into $G_{2}$. (Let the set of points of $G_{1}$ be $\{(i, j) \mid i \in Q, 1 \leq j \leq k\}$ and for each $b \in B$, make $k$ arcs $a_{b j}, j=1, \ldots, k$, of $G_{1}$ such that

$$
\left.\mathrm{i}\left(a_{b j}\right)=(\mathrm{i}(b), j), \quad \mathrm{t}\left(a_{b j}\right)=\left(\mathrm{t}(b), \mu_{b}(j)\right), \quad \text { and } h\left(a_{b j}\right)=b .\right)
$$

Thus we have the following proposition.

Proposition 2.3. Let $G=(Q, B, \zeta)$ be a strongly connected graph with $|Q|=q$ and $M(G)=\left(m_{i j}\right)$. Then $G_{1}$ is a biregular extension of $G$ iff for some positive integer $k, M\left(G_{1}\right)$ is written as a square matrix of order $q k$ of the form

$$
M\left(G_{1}\right)=\left(\begin{array}{lll}
M_{11} & \cdots & M_{1 q} \\
& \cdots & \\
M_{q 1} & \cdots & M_{q q}
\end{array}\right)
$$

where $M_{i j}$ is the sum of some $m_{i j}$ permutation matrices of order $k$.

A rectangular 0-1 matrix with non-zero columns and with exactly one 1 in each row, is called an amalgamation matrix.

Let $h$ be a homomorphism of a graph $G_{1}$ with $m$ points, $u_{1}, \ldots, u_{m}$, into a graph $G_{2}$ with $n$ points $v_{1}, \ldots, v_{n}$, and let $\phi_{h}$ be onto. Let $R$ be the $m \times n$ matrix with $R=\left(r_{i j}\right)$ where $r_{i j}=1$ if $\phi_{h}\left(u_{i}\right)=v_{j}$ and otherwise $r_{i j}=0$ for $i=1, \ldots, m$ and $j=$ $1, \ldots, n$. Clearly $R$ is an amalgamation matrix. We call $R$ the amalgamation matrix associated with $\phi_{h}$.

A similar result to the following appears in [18].

Proposition 2.4. Let $G_{1}$ and $G_{2}$ be graphs. If $h$ is a regular [b-regular] homomorphism of $G_{1}$ into $G_{2}$, then $M\left(G_{1}\right) R=R M\left(G_{2}\right)\left[R^{t} M\left(G_{1}\right)=M\left(G_{2}\right) R^{t}\right]$, where $R$ is the amalgamation matrix associated with $\phi_{h}$. [ $R^{t}$ denotes the transpose of $\left.R.\right]$ Conversely if $R$ is an amalgamation matrix satisfying $M\left(G_{1}\right) R=R M\left(G_{2}\right)\left[R^{t} M\left(G_{1}\right)=\right.$ $\left.M\left(G_{2}\right) R^{t}\right]$, then there exists a regular [b-regular] homomorphism $h$ of $G_{1}$ into $G_{2}$ such that $R$ is the amalgamation matrix associated with $\phi_{h}$.

Proof. Let $G_{1}=\left(P, A, \zeta_{1}\right)$ and $G_{2}=\left(Q, B, \zeta_{2}\right)$ with $P=\left\{u_{1}, \ldots, u_{m}\right\}$ and $Q=$ $\left\{v_{1}, \ldots, v_{n}\right\}$. For any $1 \leq i, j \leq m$, let $A_{i j}$ be the set of arcs of $G_{1}$ going from $u_{i}$ to $u_{j}$. For any $1 \leq k, l \leq n$, let $B_{k l}$ be the set of arcs of $G_{2}$ going from $v_{k}$ to $v_{l}$. 
Let $h$ be a regular homomorphism of $G_{1}$ into $G_{2}$ and let $R$ be the amalgamation matrix associated with $\phi_{h}$. Since $h$ is a regular homomorphism, for any $i, j$ with $1 \leq i \leq m$ and $1 \leq j \leq n$, we have

$$
\sum_{u_{i} \in \phi_{h}^{-1}\left(v_{j}\right)}\left|A_{i l}\right|=\left|B_{k_{i} j}\right|
$$

where $k_{i}$ is the index such that $\phi_{h}\left(u_{i}\right)=v_{k_{i}}$. Since the left-hand-side of the above equation equals the $(i, j)$ entry of $M\left(G_{1}\right) R$ and the right-hand-side equals the $(i, j)$ entry of $R M\left(G_{2}\right)$, we have $M\left(G_{1}\right) R=R M\left(G_{2}\right)$.

Conversely, assume that $R=\left(r_{i j}\right)$ is an amalgamation matrix such that $M\left(G_{1}\right) R=$ $R M\left(G_{2}\right)$. Let $\phi: P \rightarrow Q$ be the mapping such that $r_{i j}=1$ iff $\phi\left(u_{i}\right)=v_{j}, \quad(i=$ $1, \ldots, m, j=1, \ldots, n)$. Then since $M\left(G_{1}\right) R=R M\left(G_{2}\right)$, for any $i, j$ with $1 \leq i \leq m$ and $1 \leq j \leq n$, we have

$$
\sum_{u_{i} \in \phi^{-1}\left(v_{j}\right)}\left|A_{i l}\right|=\left|B_{k_{i} j}\right|
$$

where $k_{i}$ is the index such that $\phi\left(u_{i}\right)=v_{k_{i}}$. For any $i, j$ with $1 \leq i \leq m$ and $1 \leq j \leq n$, let

$$
h_{i j}: \bigcup_{u_{i} \in \phi^{-1}\left(v_{j}\right)} A_{i l} \rightarrow B_{k_{i} j}
$$

be any bijection. Let $h: A \rightarrow B$ be defined as follows: $h(a)=h_{i j}(a)$ if $a \in \bigcup_{u_{l} \in \phi^{-1}\left(v_{j}\right)} A_{i l}$ for $i=1, \ldots, m$ and $j=1, \ldots, n$. It is easy to see that $h$ is a regular homomorphism of $G_{1}$ into $G_{2}$ with $\phi_{h}=\phi$.

Proposition 2.5. Let $G_{1}$ and $G_{2}$ be graphs such that $G_{1}$ is a biregular extension of $G_{2}$. Then the elementary divisors of $M\left(G_{2}\right)$ is contained in the elementary divisors of $M\left(G_{1}\right)$.

Proof. By proposition 2.4, there is an amalgamation matrix $R$ such that

$$
M\left(G_{1}\right) R=R M\left(G_{2}\right) \text { and } R^{t} M\left(G_{1}\right)=M\left(G_{2}\right) R^{t}
$$

Since $R$ is an amalgamation matrix, the columns of $R$ are non-zero and any distinct two of them are orthogonal. Hence if $R$ is a $p \times q$ matrix, we can choose a $p \times(p-q)$ matrix $S$ with non-zero columns such that any two distinct columns of the $p \times p$ matrix $T$ of the form ( $R \quad S)$ are orthogonal. That is, $R^{t} S=0$, and $T^{t} T$ and $S^{t} S$ together with $R^{t} R$ are diagonal matrices. Therefore, since $M\left(G_{1}\right) R=R M\left(G_{2}\right)$ and $R^{t} M\left(G_{1}\right)=M\left(G_{2}\right) R^{t}$, it follows that

$$
\begin{aligned}
T^{-1} M\left(G_{1}\right) T & =\left(T^{t} T\right)^{-1} T^{t} M\left(G_{1}\right) T \\
& =\left(T^{t} T\right)^{-1}\left(\begin{array}{c}
R^{t} \\
S^{t}
\end{array}\right) M\left(G_{1}\right)(R \quad S) \\
& =\left(T^{t} T\right)^{-1}\left(\begin{array}{cc}
R^{t} M\left(G_{1}\right) R & R^{t} M\left(G_{1}\right) S \\
S^{t} M\left(G_{1}\right) R & S^{t} M\left(G_{1}\right) S
\end{array}\right) \\
& =\left(\begin{array}{cc}
\left(R^{t} R\right)^{-1} & 0 \\
0 & \left(S^{t} S\right)^{-1}
\end{array}\right)\left(\begin{array}{cc}
R^{t} R M\left(G_{2}\right) & M\left(G_{2}\right) R^{t} S \\
S^{t} R M\left(G_{2}\right) & S^{t} M\left(G_{1}\right) S
\end{array}\right) \\
& =\left(\begin{array}{cc}
M\left(G_{2}\right) & 0 \\
0 & M_{1}
\end{array}\right)
\end{aligned}
$$


where $M_{1}=\left(S^{t} S\right)^{-1} S^{t} M\left(G_{1}\right) S$. Thus the result follows. (See [6, Vol. I, Chap. VI, theorem 5.)

3. A maximal compatible set is a minimal complete set

Let $G_{1}=\left(P, A, \zeta_{1}\right)$ and $G_{2}=\left(Q, B, \zeta_{2}\right)$ be two graphs and let $h$ be a homomorphism of $G_{1}$ into $G_{2}$. Let $U \subset P$ and let $y \in \Pi\left(G_{2}\right)$. Define

$$
\mathrm{C}_{h}(U, y)=\left\{\mathrm{t}(x) \mid x \in \mathrm{\Pi}\left(G_{1}\right), \quad \mathrm{i}(x) \in U, \quad h^{*}(x)=y\right\}
$$

and

$$
\overline{\mathrm{C}}_{h}(y, U)=\left\{\mathrm{i}(x) \mid x \in \Pi\left(G_{1}\right), \quad \mathrm{t}(x) \in U, \quad h^{*}(x)=y\right\} .
$$

For $u \in P$ and $y \in \Pi\left(G_{2}\right)$, we denote $\mathrm{C}_{h}(\{u\}, y)\left[\overline{\mathrm{C}}_{h}(y,\{u\})\right]$ by $\mathrm{C}_{h}(u, y)\left[\overline{\mathrm{C}}_{h}(y, u)\right]$. A subset $U$ of $P$ is called a compatible set [a backward-compatible (abbreviated to $b$-compatible) set $]$ for $h$ if $U=\mathrm{C}_{h}(u, y)\left[U=\overline{\mathrm{C}}_{h}(y, u)\right]$ for some $u \in P$ and $y \in \Pi\left(G_{2}\right)$. A subset $U$ of $P$ is called a complete set [a backward-complete (abbreviated to $b$-complete) set] for $h$, if there exists $v \in Q$ such that $U \subset \phi_{h}^{-1}(v)$ and $C_{h}(U, y) \neq \varnothing$ $\left[\overrightarrow{\mathrm{C}}_{h}(y, U) \neq \varnothing\right]$ for all $y \in \Pi\left(G_{2}\right)$ with $\mathrm{i}(y)=v[\mathrm{t}(y)=v]$.

LEMMA 3.1. Let $G_{1}$ and $G_{2}$ be graphs and let $h$ be a homomorphism of $G_{1}$ into $G_{2}$. If $h^{*}$ is onto, then there exists a compatible [b-compatible] set for $h$ which is a complete [b-complete] set for $h$.

Proof. Assume that $h^{*}$ is onto but that any compatible set for $h$ is not a complete set for $h$. Let $v$ be a point of $G_{2}$. Since $h^{*}$ is onto, $\phi_{h}^{-1}(v)$ is a complete set for $h$. Let $\phi_{h}^{-1}(v)=\left\{u_{1}, \ldots, u_{p}\right\}$. Since a compatible set $\left\{u_{1}\right\}$ is not a complete set, there exists $y_{1} \in \Pi\left(G_{2}\right)$ such that $\mathrm{i}\left(y_{1}\right)=v$ and $\mathrm{C}\left(u_{1}, y_{1}\right)=\varnothing$. (If $h$ is understood, we shall often omit 'for $h$ ' and the suffix $h$ of $C_{h}(u, y)$.) Since a compatible set $C\left(u_{2}, y_{1}\right)$ is' not a complete set, there exists $y_{2} \in \Pi\left(G_{2}\right)$ such that $\mathrm{i}\left(y_{2}\right)=\mathrm{t}\left(y_{1}\right)$ and

$$
\mathrm{C}\left(u_{2}, y_{1} y_{2}\right)=\mathrm{C}\left(\mathrm{C}\left(u_{2}, y_{1}\right), y_{2}\right)=\varnothing \text {. }
$$

Proceeding in this way, we have $y_{1}, \ldots, y_{p}$ in $\Pi\left(G_{2}\right)$ such that $y_{1} \cdots y_{p} \in \Pi\left(G_{2}\right)$ and

$$
\mathrm{C}\left(u_{i}, y_{1} \cdots y_{i}\right)=\varnothing \text { for } i=1, \ldots, p \text {. }
$$

Hence we have

$$
\mathrm{C}\left(\phi_{h}^{-1}(v), y_{1} \cdots y_{p}\right)=\bigcup_{i=1}^{p} \mathrm{C}\left(u_{i}, y_{1} \cdots y_{p}\right)=\varnothing,
$$

which is a contradiction.

THEOREM 3.2. Let $G_{1}=\left(P, A, \zeta_{1}\right)$ and $G_{2}=\left(Q, B, \zeta_{2}\right)$ be two strongly connected graphs with $r\left(G_{1}\right)=r\left(G_{2}\right)$. Let $h$ be a homomorphism of $G_{1}$ into $G_{2}$ with $h^{*}$ onto. Then every maximal compatible [b-compatible] set for $h$ is a minimal complete [b-complete] set for $h$.

Proof. Let $U$ be a maximal compatible set for $h$. Then there exists $u \in P$ and $y \in \Pi\left(G_{2}\right)$ such that $U=\mathrm{C}(u, y)$. By lemma 3.1, there exists a complete set written as $\mathrm{C}(v, z)$ with $v \in P$ and $z \in \Pi\left(G_{2}\right)$. Let $v^{\prime} \in \mathrm{C}(v, z)$. Since $G_{1}$ is strongly connected, there exists a path $x$ going from $v^{\prime}$ to $u$. Clearly

$$
\mathrm{C}\left(\boldsymbol{v}, z h^{*}(x) y\right) \supset U \text {. }
$$


Since $U$ is a maximal compatible set, $U=\mathrm{C}\left(v, z h^{*}(x) y\right)$. Since $\mathrm{C}(v, z)$ is a complete set,

$$
U=\mathrm{C}\left(\mathrm{C}(v, z), h^{*}(x) y\right)
$$

is a complete set.

Assume that there exists $s \in U$ such that $U^{\prime}=U-\{s\}$ is a complete set for $h$. Let $C(t, w)$ be a maximal compatible set with $t \in P$ and $w \in \Pi\left(G_{2}\right)$. Since $G_{1}$ is strongly connected, there exists a path $x^{\prime}$ in $G_{1}$ going from $s$ to $t$. Clearly we have

$$
\mathrm{C}(t, w) \subset \mathrm{C}\left(s, h^{*}\left(x^{\prime}\right) w\right) \subset \mathrm{C}\left(U, h^{*}\left(x^{\prime}\right) w\right) \text {. }
$$

Since $U$ is a compatible set, $\mathrm{C}\left(U, h^{*}\left(x^{\prime}\right) w\right)$ is a compatible set. Since $\mathrm{C}(t, w)$ is a maximal compatible set, it follows that $\mathrm{C}(t, w)=\mathrm{C}\left(U, h^{*}\left(x^{\prime}\right) w\right)$, so that

$$
\mathrm{C}\left(s, h^{*}\left(x^{\prime}\right) w\right)=\mathrm{C}\left(U, h^{*}\left(x^{\prime}\right) w\right) .
$$

Since $U^{\prime}$ is a complete set, $\mathrm{C}\left(U^{\prime}, h^{*}\left(x^{\prime}\right) w\right) \neq \varnothing$. Hence there exist $s^{\prime} \in U^{\prime}$ and $p \in \mathrm{C}\left(U^{\prime}, h^{*}\left(x^{\prime}\right) w\right)$ and a path $x_{1}$ going from $s^{\prime}$ to $p$ with

$$
h^{*}\left(x_{1}\right)=h^{*}\left(x^{\prime}\right) w
$$

Since $\mathrm{C}\left(U^{\prime}, h^{*}\left(x^{\prime}\right) w\right) \subset \mathrm{C}\left(U, h^{*}\left(x^{\prime}\right) w\right)=\mathrm{C}\left(s, h^{*}\left(x^{\prime}\right) w\right), p \in \mathrm{C}\left(s, h^{*}\left(x^{\prime}\right) w\right)$. Hence there exists a path $x_{2}$ going from $s$ to $p$ with

$$
h^{*}\left(x_{2}\right)=h^{*}\left(x^{\prime}\right) w .
$$

Since $U$ is a compatible set and $s, s^{\prime} \in U$, there exist two paths $x_{3}$ and $x_{4}$ in $G_{1}$ such that

$$
\mathrm{i}\left(x_{3}\right)=\mathrm{i}\left(x_{4}\right), \quad \mathrm{t}\left(x_{3}\right)=s^{\prime}, \quad \mathrm{t}\left(x_{4}\right)=s \quad \text { and } \quad h^{*}\left(x_{3}\right)=h^{*}\left(x_{4}\right) .
$$

Hence $x_{3} x_{1}$ and $x_{4} x_{2}$ are two distinct paths in $G_{1}$ which are indistinguishable by $h$. This is a contradiction (see $\S 1$ ). Thus we conclude that $U$ is a minimal complete set.

As a corollary of the above theorem, we have the following basic result, which can be viewed as a generalization of a result of L. R. Welch, [8, theorem 14.4]. (Cf. [14, lemma 2].)

COROLLARY 3.3. Let $G_{1}$ and $G_{2}$ be two strongly connected graphs with $r\left(G_{1}\right)=r\left(G_{2}\right)$. Let $h$ be a homomorphism of $G_{1}$ into $G_{2}$ with $h^{*}$ onto. If $U$ is a maximal compatible [b-compatible] set for $h$, then for any path $y$ in $G_{2}$ with $\mathrm{i}(y) \in \phi_{h}(U)\left[\mathrm{t}(y) \in \phi_{h}(U)\right]$, $\mathrm{C}_{h}(U, y)\left[\overline{\mathrm{C}}_{h}(y, U)\right]$ is a maximal compatible [b-compatible] set for $h$.

Proof. Let $U$ be a maximal compatible set for $h$. Let $y$ be a path in $G_{2}$ with $\mathrm{i}(y) \in \phi_{h}(U)$. From theorem $3.2, U$ is a complete set. Hence $\mathrm{C}(U, y)$ is a complete set. Since $U$ is a compatible set, $\mathrm{C}(U, y)$ is a compatible set. Let $V$ be a maximal compatible set such that $V \supset \mathrm{C}(U, y)$. Then from theorem $3.2, V$ is a minimal complete set. Therefore since $\mathrm{C}(U, y)$ is a complete set, we have $V=\mathrm{C}(U, y)$. Thus $\mathrm{C}(U, y)$ is a maximal compatible set.

\section{Induced regular and b-regular homomorphisms}

By virtue of corollary 3.3, we can introduce the notions of induced regular homomorphism' and 'induced b-regular homomorphism' which are associated with 
every homomorphism $h$ between two strongly connected graphs such that $h^{*}$ is uniformly finite-to-one and onto. (These are a generalization of 'right $\lambda$-bundlegraph' and 'left $\lambda$-bundle-graph' of [14].)

Throughout this section, we assume that $G_{1}=\left(P, A, \zeta_{1}\right)$ and $G_{2}=\left(Q, B, \zeta_{2}\right)$ are two strongly connected graphs with $r\left(G_{1}\right)=r\left(G_{2}\right)$ and $h$ is a homomorphism of $G_{1}$ into $G_{2}$ such that $h^{*}$ is onto.

Denote by $\mathscr{C}_{h}\left[\overline{\mathscr{C}}_{h}\right]$ the set of all maximal compatible [b-compatible] sets for $h$. For any $U \subset P$ and $y \in \Pi\left(G_{2}\right)$, we define

$$
\mathrm{B}_{h}(U, y)=\left\{x \in \Pi\left(G_{1}\right) \mid \mathrm{i}(x) \in U, \quad h^{*}(x)=y\right\}
$$

and

$$
\overline{\mathrm{B}}_{h}(y, U)=\left\{x \in \Pi\left(G_{1}\right) \mid \mathrm{t}(x) \in U, \quad h^{*}(x)=y\right\} .
$$

We define the bundle-graph induced by $h$ as the graph

$$
\mathscr{G}_{h}=\left(\mathscr{C}_{h}, \mathscr{E}_{h}, \zeta_{h}\right)
$$

where $\mathscr{C}_{h}$ is the set of all pairs of the form $\left(U, \mathrm{~B}_{h}(U, b)\right)$ where $U \in \mathscr{C}_{h}$ and $b \in B$ with i $(b) \in \phi_{h}(U)$, and $\zeta_{h}: \mathscr{E}_{h} \rightarrow \mathscr{C}_{h} \times \mathscr{C}_{h}$ is defined as follows:

$$
\zeta_{h}\left(\left(U, \mathrm{~B}_{h}(U, b)\right)\right)=\left(U, \mathrm{C}_{h}(U, b)\right)
$$

for all $U \in \mathscr{C}_{h}$ and $b \in B$ with $\mathrm{i}(b) \in \phi_{h}(U)$. By corollary $3.3, \mathrm{C}_{h}(U, b) \in \mathscr{C}_{h}$ for any $U \in \mathscr{C}_{h}$ and $b \in B$ with $\mathrm{i}(b) \in \phi_{h}(U)$. Hence $\zeta_{h}$ is well-defined. Furthermore, we define a mapping $\tilde{h}: \mathscr{E}_{h} \rightarrow B$ as follows:

$$
\tilde{h}\left(\left(U, \mathrm{~B}_{h}(U, b)\right)\right)=b
$$

for all $U \in \mathscr{C}_{h}$ and $b \in B$ with $\mathrm{i}(b) \in \phi_{h}(U)$.

Similarly, the backward bundle-graph (abbreviated to b-bundle-graph) induced by $h$ is defined to be the graph

$$
\overline{\mathscr{G}}_{h}=\left(\overline{\mathscr{C}}_{h}, \overline{\mathscr{C}}_{h}, \bar{\zeta}_{h}\right)
$$

where $\overline{\mathscr{C}}_{h}$ is the set of all pairs of the form $\left(\overline{\mathrm{B}}_{h}(b, U), U\right)$ where $U \in \overline{\mathscr{C}}_{h}$ and $b \in B$ with $\mathbf{t}(b) \in \phi_{h}(U)$ and $\bar{\zeta}_{h}: \overline{\mathscr{C}}_{h} \rightarrow \overline{\mathscr{C}}_{h} \times \overline{\mathscr{C}}_{h}$ is defined as follows:

$$
\bar{\zeta}_{h}\left(\left(\overline{\mathrm{B}}_{h}(b, U), U\right)\right)=\left(\overline{\mathrm{C}}_{h}(b, U), U\right)
$$

for all $U \in \overline{\mathscr{C}}_{h}$ and $b \in B$ with $\mathbf{t}(b) \in \phi_{h}(U)$. Also, by virtue of corollary $3.3, \bar{\zeta}_{h}$ is well-defined. We define a mapping $\bar{h}: \overline{\mathscr{E}}_{h} \rightarrow B$ as follows:

$$
\overline{\tilde{h}}\left(\left(\overline{\mathrm{B}}_{h}(b, U), U\right)\right)=b
$$

for all $U \in \overline{\mathscr{C}}_{h}$ and $b \in B$ with $\mathrm{t}(b) \in \phi_{h}(U)$.

PROPOSITION 4.1. The bundle-graph $\mathscr{G}_{h}\left[\right.$ b-bundle-graph $\left.\overline{\mathscr{G}}_{h}\right]$ is strongly connected and the mapping $\tilde{h}[\overline{\tilde{h}}]$ is a regular [b-regular] homomorphism of $\mathscr{G}_{h}\left[\overline{\mathscr{G}}_{h}\right]$ into $G_{2}$.

Proof. Let $U, V \in \mathscr{C}_{h}$. If $\mathrm{C}(U, z)=V$ for $z=b_{1} \cdots b_{p} \in \Pi\left(G_{2}\right)$ with $b_{1}, \ldots, b_{p} \in B$, then there exists a path $E_{1} \cdots E_{p}$ in $\mathscr{G}_{h}$ such that

$$
E_{i}=\left(U_{i}, \mathrm{~B}_{h}\left(U_{i}, b_{i}\right)\right) \quad \text { for } i=1, \ldots, p
$$

where $U_{1}=U, U_{i+1}=\mathrm{C}\left(U_{i}, b_{i}\right)$ for $i=1, \ldots, p$ and $U_{p+1}=V$. Hence to prove that $\mathscr{G}_{h}$ is strongly connected, it suffices to show that there exists a path $z$ in $G_{2}$ such that $\mathrm{C}(U, z)=V$. Since $V$ is a compatible set, there exists $v \in P$ and $y \in \Pi\left(G_{2}\right)$ such 
that $V=\mathrm{C}(v, y)$. Since $G_{1}$ is strongly connected, there exists a path $x$ such that $\mathrm{i}(x) \in U$ and $\mathrm{t}(x)=v$. Clearly,

$$
C\left(U, h^{*}(x) y\right) \supset V .
$$

Since $\mathrm{C}\left(U, h^{*}(x) y\right)$ is a compatible set and $V$ is a maximal compatible set, we have

$$
\mathrm{C}\left(U, h^{*}(x) y\right)=V \text {. }
$$

The remainder is clear from the construction.

Remark 4.2. Each of $(\tilde{h})^{*}$ and $(\overline{\bar{h}})^{*}$ is uniformly finite-to-one and onto, and $r\left(\mathscr{G}_{h}\right)=r\left(\overline{\mathscr{G}}_{h}\right)=r\left(G_{2}\right)$. This follows from propositions 4.1 and 2.1 .

We call $\tilde{h}[\overline{\tilde{h}}]$ the induced regular [b-regular] homomorphism of $h$.

It follows from corollary 3.3 that each subset of paths in $G_{1}$ of the form $\mathrm{B}_{h}(U, y)$ $\left[\overline{\mathrm{B}}_{h}(y, U)\right]$ where $U \in \mathscr{C}_{h}\left[U \in \overline{\mathscr{C}}_{h}\right]$ and $y \in \Pi\left(G_{2}\right)$ with i $(y) \in \phi_{h}(U)\left[\mathrm{t}(y) \in \phi_{h}(U)\right]$, is non-empty. To each path $Z$ of length $p \geq 0$ in $\mathscr{G}_{h}\left[\overline{\mathscr{G}}_{h}\right]$ corresponds the non-empty subset of paths $\mathrm{B}_{h}(U, y)\left[\overline{\mathrm{B}}_{h}(y, U)\right]$ of length $p$ in $G_{1}$ where $\mathrm{i}(Z)=U[\mathrm{t}(Z)=U]$ and $y=\tilde{h}(Z)[y=\tilde{h}(Z)]$. It is called the bundle of $Z$ and is denoted by $\mathrm{B}(Z)$. Clearly each subset of paths in $G_{1}$ of the form $\mathrm{B}_{h}(U, y)\left[\widetilde{B}_{h}(y, U)\right]$ where $U \in$ $\mathscr{C}_{h}\left[U \in \overline{\mathscr{C}}_{h}\right]$ and $y \in \Pi^{(p)}\left(G_{2}\right)$ with $\mathrm{i}(y) \in \phi_{h}(U)\left[\mathrm{t}(y) \in \phi_{h}(U)\right]$, is the bundle of some path of length $p$ in $\mathscr{G}_{h}\left[\overline{\mathscr{G}}_{h}\right]$, and is also called a bundle [backward bundle, abbreviated to $b$-bundle] of length $p$ for $h$.

For $\Gamma \in \Omega\left(\mathscr{G}_{h}\right)\left[\Gamma \in \Omega\left(\overline{\mathscr{G}}_{h}\right)\right]$ and $\alpha \in \Omega\left(G_{1}\right)$, we say that $\Gamma$ contains $\alpha$ if $\mathrm{B}\left(\Gamma_{i}\right) \ni \alpha_{i}$ for all $i \in \mathbb{Z}$.

LEMMA 4.3. For each $\Gamma \in \Omega\left(\mathscr{G}_{h}\right)\left[\Gamma \in \Omega\left(\overline{\mathscr{G}}_{h}\right)\right]$, there exists $\alpha \in \Omega\left(G_{1}\right)$ such that $\Gamma$ contains $\alpha$, and for each $\alpha \in \Omega\left(G_{1}\right)$, there exists $\Gamma \in \Omega\left(\mathscr{G}_{h}\right)\left[\Gamma \in \Omega\left(\overline{\mathscr{G}}_{h}\right)\right]$ such that $\Gamma$ contains $\alpha$.

Proof. Let $\Gamma \in \Omega\left(\mathscr{G}_{h}\right)$. For each non-negative integer $k$, there exists an element $x_{k}$ of $\mathrm{B}(\Gamma[-k, k])$, and there exists $\alpha^{(k)} \in \Omega\left(G_{1}\right)$ such that

$$
\alpha^{(k)}[-k, k]=x_{k}
$$

(because any point of $G_{1}$ has an arc going from it and an arc going to it by our standing hypothesis for graphs). Since $\Omega\left(G_{1}\right)$ is a compact metric space, there exists a sequence $0 \leq k_{0} \leq k_{1}<\cdots$ of integers and $\alpha \in \Omega\left(G_{1}\right)$ such that

$$
\lim _{j \rightarrow \infty} \alpha^{\left(k_{j}\right)}=\alpha \text {. }
$$

It is easy to see that $\Gamma$ contains $\alpha$.

Conversely, let $\alpha \in \Omega\left(G_{1}\right)$. Let $k$ be any non-negative integer. Let $U_{k}$ be a maximal compatible set for $h$ such that $U_{k} \ni \mathrm{i}(\alpha[-k, k])$. Since $\tilde{h}$ is regular, there exists $Z_{k} \in \Pi^{(2 k+1)}\left(\mathscr{G}_{h}\right)$ going from $U_{k}$ with $\tilde{h}^{*}\left(Z_{k}\right)=h^{*}(\alpha[-k, k])$. Clearly $\mathrm{B}\left(Z_{k}\right) \ni$ $\alpha[-k, k]$. There exists $\Gamma^{(k)} \in \Omega\left(\mathscr{G}_{h}\right)$ such that $\Gamma^{(k)}[-k, k]=Z_{k}$ (because, by proposition 4.1 , any point of $\mathscr{G}_{h}$ has an arc going from it and an arc going to it). Since $\Omega\left(\mathscr{G}_{h}\right)$ is a compact metric space, there exists a sequence $0 \leq k_{0}<k_{1}<\cdots$ of integers and $\Gamma \in \Omega\left(\mathscr{G}_{h}\right)$ such that

$$
\lim _{j \rightarrow \infty} \Gamma^{\left(k_{j}\right)}=\Gamma .
$$

It is also easy to see that $\Gamma$ contains $\alpha$. 


\section{Mergible homomorphisms}

For paths $x$ and $y$ in a graph $G, y$ is an initial subpath [a terminal subpath] of $x$, if there exists a path $w$ in $G$ such that $x=y w[x=w y]$. (Here we assume that $\mathrm{i}(x) x=x \mathrm{t}(x)=x$ for each path $x$ in $G$.)

Let $h$ be a homomorphism of a graph $G_{1}$ into a graph $G_{2}$. Let $p$ be a non-negative integer. We say that $h$ is $p$ bundle-mergible [ $p$ backward-bundle-mergible (abbreviated to $p b$-bundle-mergible)] if for any two paths $x_{1}$ and $x_{2}$ of length $l \geq p$ in $G_{1}$, if $\mathrm{i}\left(x_{1}\right)=\mathrm{i}\left(x_{2}\right)\left[\mathrm{t}\left(x_{1}\right)=\mathrm{t}\left(x_{2}\right)\right]$ and $h^{*}\left(x_{1}\right)=h^{*}\left(x_{2}\right)$, then $x_{1}$ and $x_{2}$ have the same initial [terminal] subpath of length $l-p$. We say that $h$ is mergible, if for some non-negative integers $p$ and $q, h$ is both $p$ bundle-mergible and $q$ b-bundle-mergible. (The notion of ' $p$ bundle-[b-bundle]-mergible' corresponds to 'nonexistence of a right [left] $f$-branch of length $p$ ' in [8].)

Remark 5.1. Let $G_{1}$ and $G_{2}$ be strongly connected graphs with $r\left(G_{1}\right)=r\left(G_{2}\right)$, and let $h$ be a homomorphism of $G_{1}$ into $G_{2}$. Then $h$ is $p$ bundle-mergible [ $p$ b-bundlemergible] iff $h^{*}$ is onto and each bundle [b-bundle] $X$ of length $l \geq p$ for $h$, all paths in $X$ have the same initial [terminal] subpath of length $l-p$.

Proof. If $h$ is $p$ bundle-mergible, then $h^{*}$ is onto because no two distinct paths in $G_{1}$ are indistinguishable by $h$. Hence $h^{*}$ is onto (see $\S 1$ ). The proof of the remainder is straightforward.

Remark 5.2. Let $G_{1}$ and $G_{2}$ be two strongly connected graphs with $r\left(G_{1}\right)=r\left(G_{2}\right)$ and let $h$ be a homomorphism of $G_{1}$ into $G_{2}$. Then $h$ is 0 bundle-mergible [0 b-bundle-mergible] iff $h$ is regular [b-regular].

Proof. Assume that $h$ is 0 bundle-mergible. Then $h^{*}$ is onto by remark 5.1. Since $h$ is 0 bundle-mergible, it follows that for each point $u$ of $G_{2},\{u\}$ is a maximal compatible set for $h$, and for each arc $b$ with $\mathrm{i}(b)=\phi_{h}(u)$, the arc $a$ such that $\mathrm{i}(a)=u$ and $h(a)=b$, is unique; such an arc $a$ always exists because $\{u\}$ is a complete set for $h$ by theorem 3.2. Thus $h$ is regular. The converse is clear.

The terminology of $p$ bundle-mergible [ $p$ b-bundle-mergible] is based on remark 5.1. Another restatement of the property of being $p$ bundle-mergible [ $p$ b-bundlemergible], is given as the following lemma. (This can be considered as a generalization of [8, theorem 16.9].)

LEMMA 5.3. Let $G_{1}$ and $G_{2}$ be two strongly connected graphs with $r\left(G_{1}\right)=r\left(G_{2}\right)$ and let $h$ be a homomorphism of $G_{1}$ into $G_{2}$. Let $p$ be a non-negative integer. Then $h$ is $p$ bundle-mergible [p b-bundle-mergible] iff $h^{*}$ is onto and for each point $u$ of $G_{1}$ and each path $y$ of length at least $p$ in $G_{2}$ with $\mathrm{i}(y)=\phi_{h}(u)\left[\mathrm{t}(y)=\phi_{h}(u)\right]$, $\mathrm{C}_{h}(u, y)\left[\overline{\mathrm{C}}_{h}(y, u)\right]$ is either empty or a maximal compatible [b-compatible] set.

Proof. Assume that $h$ is $p$ bundle-mergible. By remark 5.1, $h^{*}$ is onto. Let $u$ be a point of $G_{1}$ and let $y$ be a path of length $l \geq p$ in $G_{2}$ with $\mathrm{i}(y)=\phi_{h}(u)$. Suppose that $\mathrm{C}(u, y) \neq \varnothing$. Let $U$ be a maximal compatible set which contains $u$. Then by corollary $3.3, \mathrm{C}(U, y)$ is a maximal compatible set. Therefore it suffices to show that $\mathrm{C}(u, y)=\mathrm{C}(U, y)$. 
Clearly $\mathrm{C}(u, y) \subset \mathrm{C}(U, y)$. Let $v$ be an arbitrary element of $\mathrm{C}(U, y)$. Then there exists $x_{1} \in \Pi\left(G_{1}\right)$ such that

$$
\mathrm{i}\left(x_{1}\right) \in U, \quad \mathrm{t}\left(x_{1}\right)=v, \quad \text { and } \quad h^{*}\left(x_{1}\right)=y .
$$

Since $\mathrm{C}(u, y) \neq \varnothing$, there exists $x_{2} \in \Pi\left(G_{1}\right)$ such that $\mathrm{i}\left(x_{2}\right)=u$ and $h^{*}\left(x_{2}\right)=y$. Clearly $x_{1}, x_{2} \in \mathrm{B}_{h}(U, y)$. Since $h$ is $p$ bundle-mergible, all paths in $\mathrm{B}_{h}(U, y)$ have the same initial subpath of length $l-p$ (remark 5.1). Hence

$$
\mathrm{i}\left(x_{1}\right)=\mathrm{i}\left(x_{2}\right)=u
$$

so that $v \in \mathrm{C}(u, y)$. Hence we have $\mathrm{C}(u, y) \supset \mathrm{C}(U, y)$. Thus $\mathrm{C}(u, y)=\mathrm{C}(U, y)$. The proof of the converse is omitted (because this will not be used in this paper).

LEMMA 5.4. Let $G_{1}$ and $G_{2}$ be two strongly connected graphs with $r\left(G_{1}\right)=r\left(G_{2}\right)$, and let $h$ be a homomorphism of $G_{1}$ into $G_{2}$. If $h$ is $p$ bundle-mergible and $q$ b-bundle-mergible, then the induced regular homomorphism $\tilde{h}$ of $h$ is 0 bundle-mergible and $p+q b$-bundle-mergible.

Proof. Since $\tilde{h}$ is regular, $\tilde{h}$ is 0 bundle-mergible (remark 5.2). Let $Z_{1}$ and $Z_{2}$ be paths in $\mathscr{G}_{h}$ such that $\mathrm{t}\left(Z_{1}\right)=\mathrm{t}\left(Z_{2}\right), \tilde{h}^{*}\left(Z_{1}\right)=\tilde{h}^{*}\left(Z_{2}\right)$, and $Z_{1}$ and $Z_{2}$ are of length $l$ with $l \geq p+q$. To show that $\tilde{h}$ is $p+q$ b-bundle mergible, we shall show that $Z_{1}$ and $Z_{2}$ have the same terminal subpath of length $l-(p+q)$. To show this, it suffices to prove that the initial subpaths of length $p+q$ of $Z_{1}$ and $Z_{2}$, say $Z_{1}^{(p+q)}$ and $Z_{2}^{(p+q)}$ respectively, have the same terminal endpoint, because $\tilde{h}$ is regular.

Let $U_{1}=\mathrm{i}\left(Z_{1}\right)$ and let $U_{2}=\mathrm{i}\left(Z_{2}\right)$. Let $y=\tilde{h}^{*}\left(Z_{1}\right)=\tilde{h}^{*}\left(Z_{2}\right)$ and write $y=b_{1} \cdots b_{l}$ where $b_{1}, \ldots, b_{l}$ are arcs of $G_{2}$. Then

$$
\begin{aligned}
& \mathrm{C}_{h}\left(U_{1}, b_{1} \cdots b_{p+q}\right)=\mathrm{t}\left(Z_{1}^{(p+q)}\right), \\
& \mathrm{C}_{h}\left(U_{2}, b_{1} \cdots b_{p+q}\right)=\mathrm{t}\left(Z_{2}^{(p+q)}\right),
\end{aligned}
$$

and

$$
\mathrm{C}_{h}\left(U_{1}, y\right)=\mathrm{t}\left(Z_{1}\right)=\mathrm{t}\left(Z_{2}\right)=\mathrm{C}_{h}\left(U_{2}, y\right) .
$$

Let $v \in \mathrm{C}_{h}\left(U_{1}, y\right)=\mathrm{C}_{h}\left(U_{2}, y\right)$. Then there are paths $x_{1}$ and $x_{2}$ in $G_{1}$ such that

$$
\mathrm{i}\left(x_{1}\right) \in U_{1}, \quad \mathrm{i}\left(x_{2}\right) \in U_{2}, \quad \mathrm{t}\left(x_{1}\right)=\mathrm{t}\left(x_{2}\right)=v, \quad \text { and } \quad h^{*}\left(x_{1}\right)=h^{*}\left(x_{2}\right)=y .
$$

Let $x_{1}^{(q)}$ and $x_{2}^{(q)}$ be the initial subpaths of length $q$ of $x_{1}$ and $x_{2}$, respectively. Since $h$ is $q$ b-bundle-mergible, $x_{1}$ and $x_{2}$ have the same terminal subpath of length $l-q$ so that

$$
\mathrm{t}\left(x_{1}^{(q)}\right)=\mathrm{t}\left(x_{2}^{(q)}\right) .
$$

Let $s=\mathrm{t}\left(x_{1}^{(q)}\right)=\mathrm{t}\left(x_{2}^{(q)}\right)$. Then $\mathrm{C}_{h}\left(s, b_{q+1} \cdots b_{p+q}\right)$ is not empty because it contains the terminal endpoint of the initial subpath of length $p+q$ of $x_{1}$. Since $h$ is $p$ bundle-mergible, it follows from lemma 5.3 that $C_{h}\left(s, b_{q+1} \cdots b_{p+q}\right)$ is a maximal compatible set. Since $U_{1}$ and $U_{2}$ are compatible sets for $h$, so are $C_{h}\left(U_{1}, b_{1} \cdots b_{p+q}\right)$ and $\mathrm{C}_{h}\left(U_{2}, b_{1} \cdots b_{p+q}\right)$. Moreover $\mathrm{C}_{h}\left(U_{1}, b_{1} \cdots b_{p+q}\right)$ and $\mathrm{C}_{h}\left(U_{2}, b_{1} \cdots b_{p+q}\right)$ contain the maximal compatible set $\mathrm{C}_{h}\left(s, b_{q+1} \cdots b_{p+q}\right)$. Therefore

$$
\mathrm{C}_{h}\left(U_{1}, b_{1} \cdots b_{p+q}\right)=\mathrm{C}_{h}\left(s, b_{q+1} \cdots b_{p+q}\right)=\mathrm{C}_{h}\left(U_{2}, b_{1} \cdots b_{p+q}\right) .
$$

Thus we have $\mathrm{t}\left(Z_{1}^{(p+q)}\right)=\mathrm{t}\left(Z_{2}^{(p+q)}\right)$. 
LEMMA 5.5. Let $G_{1}$ and $G_{2}$ be two strongly connected graphs with $r\left(G_{1}\right)=r\left(G_{2}\right)$ and let $h$ be a homomorphism of $G_{1}$ into $G_{2}$. If $h$ is $p$ bundle-mergible [ $p$ b-bundlemergible] for a non-negative integer $p$, then there exists an admissible $(p+1)$-block map

$$
f: \Pi^{(p+1)}\left(\mathscr{G}_{h}\right) \rightarrow \Pi^{(1)}\left(G_{1}\right)
$$

$\left[f: \Pi^{(p+1)}\left(\overline{\mathscr{G}}_{h}\right) \rightarrow \Pi^{(1)}\left(G_{1}\right)\right]$ such that

$$
\tilde{h}_{\infty}=h_{\infty} f_{\infty}
$$

$\left[\overline{\tilde{h}}_{\infty}=h_{\infty} f_{\infty} \sigma^{-p}\right]$ and $f_{\infty}$ is one-to-one and onto [where $\sigma: \Omega\left(\overline{\mathscr{G}}_{h}\right) \rightarrow \Omega\left(\overline{\mathscr{G}}_{h}\right)$ is the shift $]$.

Proof. Since $h$ is $p$ bundle-mergible, it follows from remark 5.1 that for each $Z \in \Pi^{(p+1)}\left(\mathscr{G}_{h}\right)$, all paths in $\mathrm{B}(Z)$ have the same initial subpath of length 1 . (Recall that $\mathrm{B}(Z)$ is the bundle of $Z$ (cf. $\S 4$ ).) Hence we can define a block map

$$
f: \Pi^{(p+1)}\left(\mathscr{G}_{h}\right) \rightarrow \Pi^{(1)}\left(G_{1}\right)
$$

as follows. For each $Z \in \Pi^{(p+1)}\left(\mathscr{G}_{h}\right), f(Z)$ is the initial subpath of length 1 of the paths in $\mathrm{B}(Z)$. It is straightforward to see that $f$ is an admissible $(p+1)$-block map and for each $\Gamma \in \Omega\left(\mathscr{G}_{h}\right), f_{\infty}(\Gamma)$ is a unique bisequence which is contained in $\Gamma$. Hence we have $\tilde{h}_{\infty}(\Gamma)=h_{\infty}\left(f_{\infty}(\Gamma)\right)$ for each $\Gamma \in \Omega\left(\mathscr{G}_{h}\right)$ and it suffices to show that for any $\alpha \in \Omega\left(G_{1}\right)$, there exists a unique element $\Gamma$ of $\Omega\left(\mathscr{G}_{h}\right)$ which contains $\alpha$.

Let $\alpha \in \Omega\left(G_{1}\right)$. By lemma 4.3 , there exists $\Gamma \in \Omega\left(\mathscr{G}_{h}\right)$ such that $\Gamma$ contains $\alpha$. Suppose that $\Gamma^{\prime}$ in $\Omega\left(\mathscr{G}_{h}\right)$ contains $\alpha$. Let $i \in \mathbb{Z}$. If $p=0$, then $h$ is regular. Hence each maximal compatible set for $h$ consists of a single point of $G_{1}$. Hence

$$
\mathrm{i}\left(\Gamma_{i}\right)=\mathrm{i}\left(\alpha_{i}\right)=\mathrm{i}\left(\Gamma_{i}^{\prime}\right) \text {. }
$$

Assume that $p \geq 1$. Since $\mathrm{i}\left(\Gamma_{i-p}\right) \ni \mathrm{i}\left(\alpha_{i-p}\right)$,

$$
\mathrm{t}\left(\Gamma_{i-1}\right)=\mathrm{C}_{h}\left(\mathrm{i}\left(\Gamma_{i-p}\right), h^{*}(\alpha[i-p, i-1])\right) \supset \mathrm{C}_{h}\left(\mathrm{i}\left(\alpha_{i-p}\right), h^{*}(\alpha[i-p, i-1])\right) .
$$

Since $h$ is $p$ bundle-mergible, it follows from lemma 5.3 that

$$
\mathrm{C}_{h}\left(\mathrm{i}\left(\alpha_{i-p}\right), h^{*}(\alpha[i-p, i-1])\right)
$$

is a maximal compatible set. Since $\mathrm{t}\left(\Gamma_{i-1}\right)$ is a compatible set for $h$, we have

$$
\mathrm{t}\left(\Gamma_{i-1}\right)=\mathrm{C}_{h}\left(i\left(\alpha_{i-p}\right), h^{*}(\alpha[i-p, i-1])\right) .
$$

For the same reason,

$$
\mathrm{t}\left(\Gamma_{i-1}^{\prime}\right)=\mathrm{C}_{h}\left(\mathrm{i}\left(\alpha_{i-p}\right), h^{*}(\alpha[i-p, i-1])\right) .
$$

Hence we have

$$
\mathrm{i}\left(\Gamma_{i}\right)=\mathrm{t}\left(\Gamma_{i-1}\right)=\mathrm{t}\left(\Gamma_{i-1}^{\prime}\right)=\mathrm{i}\left(\Gamma_{i}^{\prime}\right) .
$$

Since $\tilde{h}\left(\Gamma_{i}\right)=h\left(\alpha_{i}\right)=\tilde{h}\left(\Gamma_{i}^{\prime}\right)$ and $\tilde{h}$ is a regular homomorphism, we have $\Gamma_{i}=\Gamma_{i}^{\prime}$. Since $i$ was arbitrary, we have $\Gamma=\Gamma^{\prime}$.

Recently, the author learned that in [11], Kitchens has a similar result to lemma 5.5.

Let $G$ be a graph and let $n$ be a non-negative integer. We consider the path graph

$$
L^{(n)}(G)=\left(\Pi^{(n)}(G), \Pi^{(n+1)}(G), \zeta^{(n)}\right)
$$

of length $n$ of $G$ (cf. $\S 1$ ). For each path $x$ of length at least $n$ in $G$, we define $(x)_{n}$ as follows. If $x$ is of length $n$, then $(x)_{n}=x$, and if $x=a_{1} \cdots a_{l}$ where $l \geq n+1$ and $a_{1}, \ldots, a_{l}$ are arcs of $G$, then

$$
(x)_{n}=\left(a_{1} \cdots a_{n+1}\right)\left(a_{2} \cdots a_{n+2}\right) \cdots\left(a_{l-n} \cdots a_{l}\right) .
$$


Then if $x$ is a path of length $l \geq n$ in $G$, then $(x)_{n}$ is a path of length $l-n$ in $L^{(n)}(G)$. Obviously, each path in $L^{(n)}(G)$ is written as $(x)_{n}$ for some path $x$ of length at least $n$ in $G$.

Let $h$ be a homomorphism of a graph $G_{1}$ into a graph $G_{2}$. Let $n$ be a non-negative integer. We define a mapping

$$
h^{(n)}: \Pi^{(n+1)}\left(G_{1}\right) \rightarrow \Pi^{(n+1)}\left(G_{2}\right)
$$

by

$$
h^{(n)}(x)=h^{*}(x) \quad x \in \Pi^{(n+1)}\left(G_{1}\right) .
$$

Clearly $h^{(n)}$ is a homomorphism of $L^{(n)}\left(G_{1}\right)$ into $L^{(n)}\left(G_{2}\right)$ and for each path $x$ of length at least $n$ in $G$,

$$
\left(h^{(n)}\right)^{*}\left((x)_{n}\right)=\left(h^{*}(x)\right)_{n}
$$

One readily gets the following.

LEMMA 5.6. Let $h$ be a homomorphism of a graph $G_{1}$ into a graph $G_{2}$, and let $n$ and $p$ be non-negative integers. If $h$ is $p$ bundle-mergible [ $p$ b-bundle-mergible], then $h^{(n)}$ is a p bundle-mergible [p b-bundle-mergible] homomorphism of $L^{(n)}\left(G_{1}\right)$ into $L^{(n)}\left(G_{2}\right)$.

LEMMA 5.7. Let $G_{1}$ and $G_{2}$ be two strongly connected graphs with $r\left(G_{1}\right)=r\left(G_{2}\right)$ and let $h$ be a homomorphism of $G_{1}$ into $G_{2}$. Assume that $h$ is $p$ bundle-mergible [ $p$ $b$-bundle-mergible] for a non-negative integer $p$. Then any two distinct maximal compatible [b-compatible] sets for $h^{(p)}$ are disjoint.

Proof. First we note that $L^{(p)}\left(G_{1}\right)$ and $L^{(p)}\left(G_{2}\right)$ are strongly connected and

$$
r\left(L^{(p)}\left(G_{1}\right)\right)=r\left(G_{1}\right)=r\left(G_{2}\right)=r\left(L^{(p)}\left(G_{2}\right)\right) .
$$

From lemma $5.6, h^{(p)}$ is $p$ bundle-mergible. Let $W$ be a maximal compatible set for $h^{(p)}$. Then since $h^{(p)}$ is $p$ bundle-mergible, it follows from lemma 5.3 that there exists a point $w \in \Pi^{(p)}\left(G_{1}\right)$ of $L^{(p)}\left(G_{1}\right)$ and a path $s$ of length $p$ in $L^{(p)}\left(G_{2}\right)$ such that

$$
W=\mathrm{C}_{h^{(p)}}(w, s) \text {. }
$$

(There exists $z \in \Pi\left(L^{(p)}\left(G_{1}\right)\right)$ such that

$$
W=\mathrm{C}_{h^{(p)}}\left(\mathrm{i}(z),\left(h^{(p)}\right)^{*}(z)\right) \text {. }
$$

We may assume that the length of $z$ is not less than $p$. Let $\hat{z}$ be the terminal subpath of length $p$ of $z$. Then since $h^{(p)}$ is $p$ bundle-mergible, it follows from lemma 5.3 that

$$
W=\mathrm{C}_{h^{(p)}\left(\mathrm{i}(\hat{z}),\left(h^{(p)}\right)^{*}(\hat{z})\right) .}
$$

Put $w=\mathrm{i}(\hat{z})$ and put $s=\left(h^{(p)}\right)^{*}(\hat{z})$.)

There exists $y \in \Pi^{(2 p)}\left(G_{2}\right)$ such that $(y)_{p}=s$. It is straightforward to see that

$$
\mathrm{C}_{h^{(p)}}(w, s)=\left\{x \in \Pi^{(p)}\left(G_{1}\right) \mid w x \in \Pi^{(2 p)}\left(G_{1}\right), \quad h^{*}(w x)=y\right\} .
$$

This implies that if $x \in W$, then we can write $W=\mathrm{B}_{h}\left(\{\mathrm{i}(x)\}, h^{*}(x)\right)$. Thus we conclude that if $W_{1}$ and $W_{2}$ are maximal compatible sets for $h^{(p)}$ and $W_{1} \cap W_{2} \neq \varnothing$, then $W_{1}=W_{2}$. Hence any two distinct maximal compatible sets for $h^{(p)}$ are disjoint. 
LEMMA 5.8. Let $G_{1}$ and $G_{2}$ be two strongly connected graphs and let $h$ be a regular homomorphism of $G_{1}$ into $G_{2}$. If every two distinct maximal b-compatible sets for $h$ are disjoint, then the induced b-regular homomorphism $\overline{\tilde{h}}$ is biregular.

Proof. By proposition 4.1, it suffices to show that $\overline{\tilde{h}}$ is regular. Let $U$ be any point of $\overline{\mathscr{G}}_{h}$ (i.e. any maximal b-compatible set for $h$ ). Let $v=\phi_{\bar{h}}(U)$ and let $b$ be any arc of $G_{2}$ going from $v$. Let $u \in U$. Then, $\phi_{h}(u)=v$. Since $h$ is regular, there exists an arc $a$ of $G_{1}$ such that $\mathrm{i}(a)=u$ and $h(a)=b$. Let $V$ be a maximal b-compatible set for $h$ which contains $\mathrm{t}(a)$. Let $U^{\prime}=\overline{\mathrm{C}}_{h}(b, V)$ and let $E=\left(\overline{\mathrm{B}}_{h}(b, V), V\right)$. Then by definition, $E$ is an arc of $\overline{\mathscr{G}}_{h}$ going from $U^{\prime}$ to $V$ with $\overline{\tilde{h}}(E)=b$. Since $V \ni t(a)$ and $h(a)=b$,

$$
\overline{\mathrm{C}}_{h}(b, V) \ni \mathrm{i}(a)=u \text {. }
$$

Hence $U \cap U^{\prime} \ni u$. Since $U$ and $U^{\prime}$ are maximal b-compatible sets and $U \cap U^{\prime} \neq \varnothing$, it follows from the assumption of the lemma that $U=U^{\prime}$. Thus $E$ is an arc of $\overline{\mathscr{G}}_{h}$ going from $U$ and $\overline{\tilde{h}}(E)=b$.

Assume that there exists an arc $E^{\prime}$ of $\overline{\mathscr{G}}_{h}$ with $E^{\prime} \neq E$ such that $\mathrm{i}\left(E^{\prime}\right)=U$, and $\overline{\tilde{h}}\left(E^{\prime}\right)=b$. Then there exists a maximal b-compatible set $V^{\prime}$ with $V^{\prime} \neq V$ such that $E^{\prime}=\left(\overline{\mathrm{B}}_{h}\left(b, V^{\prime}\right), V^{\prime}\right)$. Since $\overline{\mathrm{C}}_{h}\left(b, V^{\prime}\right)=\mathrm{i}\left(E^{\prime}\right)=U$, there exists an arc $a^{\prime}$ of $G_{1}$ such that

$$
\mathrm{i}\left(a^{\prime}\right)=u, \quad h\left(a^{\prime}\right)=b, \quad \text { and } \quad \mathrm{t}\left(a^{\prime}\right) \dot{\epsilon} V^{\prime} .
$$

Since $V^{\prime}$ and $V$ are distinct maximal b-compatible sets for $h, V^{\prime} \cap V=\varnothing$ so that $a^{\prime} \neq a$. But this is impossible because $h$ is regular. Thus $E$ is a unique arc of $\overline{\mathscr{G}}_{h}$ with $\mathrm{i}(E)=U$ and $\overline{\tilde{h}}(E)=b$. We have proved that $\overline{\tilde{h}}$ is regular.

THEOREM 5.9. Let $G_{1}$ and $G_{2}$ be strongly connected graphs with $r\left(G_{1}\right)=r\left(G_{2}\right)$, and let $h$ be a mergible homomorphism of $G_{1}$ into $G_{2}$. Then there exist a strongly connected graph $H$, an integer $p \geq 0$, a biregular homomorphism $g$ of $H$ into $L^{(p)}\left(G_{2}\right)$ and an isomorphism $\rho:\left(\Omega\left(G_{1}\right), \sigma_{1}\right) \rightarrow(\Omega(H), \sigma)$ such that

$$
h_{\infty}=\left(h_{G_{2}, p+1,1}\right)_{\infty} g_{\infty} \rho \text {. }
$$

Proof. Let $G_{3}=\mathscr{G}_{h}$ and let $g_{1}=\tilde{h}$. Then from proposition 4.1 and remark $4.2, G_{3}$ is a strongly connected graph with $r\left(G_{3}\right)=r\left(G_{2}\right)$, and $h_{1}$ is a regular homomorphism of $G_{3}$ into $G_{2}$. Since $h$ is mergible, it follows from lemma 5.4 that $h_{1}$ is 0 bundlemergible and there exists a non-negative integer $p$ such that $h_{1}$ is $p$ b-bundlemergible. Moreover, it follows from lemma 5.5 and theorem 1.5 that there exists an isomorphism $\rho^{\prime}$ of $\left(\Omega\left(G_{3}\right), \sigma_{3}\right)$ onto $\left(\Omega\left(G_{1}\right), \sigma_{1}\right)$ such that

$$
\left(h_{1}\right)_{\infty}=h_{\infty} \rho^{\prime} \text {. }
$$

Let $G_{4}=L^{(p)}\left(G_{3}\right)$, let $G_{5}=L^{(p)}\left(G_{2}\right)$, and let $h_{2}=h_{1}^{(p)}$. Then $G_{4}$ and $G_{5}$ are strongly connected graphs with

$$
r\left(G_{4}\right)=r\left(G_{3}\right)=r\left(G_{2}\right)=r\left(G_{5}\right)
$$

and $h_{2}$ is a homomorphism of $G_{4}$ into $G_{5}$. Since $h_{1}$ is 0 bundle-mergible and $p$ b-bundle-mergible, it follows from lemma 5.6 that $h_{2}$ is 0 bundle-mergible and $p$ b-bundle-mergible. Moreover, from lemma 5.7, any two distinct maximal b-compatible sets for $h_{2}$ are disjoint. Let $\rho_{1}=\left(h_{G_{3}, p+1,1}\right)_{\infty}$ and let $\rho_{2}=\left(h_{G_{2}, p+1,1}\right)_{\infty}$ (cf. $\left.\S 1\right)$. 
Then $\rho_{1}$ is an isomorphism of $\left(\Omega\left(G_{4}\right), \sigma_{4}\right)$ onto $\left(\Omega\left(G_{3}\right), \sigma_{3}\right)$ (and $\rho_{2}$ is an isomorphism of $\left(\Omega\left(G_{5}\right), \sigma_{5}\right)$ onto $\left.\left(\Omega\left(G_{2}\right), \sigma_{2}\right)\right)$ and we have

$$
\rho_{2}\left(h_{2}\right)_{\infty}=\left(h_{1}\right)_{\infty} \rho_{1} \text {. . }
$$

Let $H=\overline{\mathscr{G}}_{h_{2}}$ and let $g=\overline{\tilde{h}}_{2}$. Then, by proposition $4.1, H$ is a strongly connected graph and $g$ is a homomorphism of $H$ into $G_{5}$. Since $h_{2}$ is regular (because $h_{2}$ is 0 bundle-mergible (remark 5.2)) and any two distinct maximal b-compatible sets for $h_{2}$ are disjoint, it follows from lemma 5.8 that $g$ is biregular. Since $h_{2}$ is $p$ b-bundlemergible, it follows from lemma 5.5 and theorem 1.5 that there exists an isomorphism $\rho^{\prime \prime}$ of $(\Omega(H), \sigma)$ onto $\left(\Omega\left(G_{4}\right), \sigma_{4}\right)$ such that

Thus we have

$$
g_{\infty}=\left(h_{2}\right)_{\infty} \rho^{\prime \prime}
$$

$$
h_{\infty} \rho^{\prime} \rho_{1} \rho^{\prime \prime}=\rho_{2} g_{\infty} .
$$

Put $\rho=\left(\rho^{\prime} \rho_{1} \rho^{\prime \prime}\right)^{-1}$. Then $\rho$ is an isomorphism of $\left(\Omega\left(G_{1}\right), \sigma_{1}\right)$ onto $(\Omega(H), \sigma)$ and we have

$$
h_{\infty}=\left(h_{G_{2}, p+1,1}\right)_{\infty} g_{\infty} \rho
$$

\section{Characterizations of constant-to-one and onto global maps}

In $[8, \S 9-\$ 12]$ Hedlund describes the properties of inverses of onto endomorphisms of full shift dynamical systems. With minor modifications in the statements and the proofs, many of them are extended to onto global maps of homomorphisms between strongly connected graphs whose adjacency matrices have the same characteristic value. (Extensions of them to onto endomorphisms of irreducible subshifts of finite type were pointed out by Coven and Paul [3], and extensions of them to finite-to-one and onto homomorphisms between TPPD sofic systems were mentioned in [4].) Many of Hedlund's discussions on a block map $f: A^{n} \rightarrow A$, where $A$ is a non-empty finite set of symbols and $n$ is a positive integer, and the mapping $f_{\infty}: \Omega_{A} \rightarrow \Omega_{A}$ defined by

$$
\left(f_{\infty}(\alpha)\right)_{i}=f\left(\alpha_{i} \alpha_{i+1} \cdots \alpha_{i+n-1}\right) \quad \alpha \in \Omega(A), i \in \mathbb{Z},
$$

can be interpreted naturally as discussions on the homomorphism $h_{f}$ of $L^{(n-1)}\left(G_{0}(A)\right)$ into $G_{0}(A)$ defined by $h_{f}(x)=f(x), x \in A^{n}$, and its global map $\left(h_{f}\right)_{\infty}$. (Cf. $\S 1$. Note that $L^{(n-1)}\left(G_{0}(A)\right)$ has point set $A^{n-1}$ and arc set $A^{n}$. Hence, for example, 'totally $(n-1)$-separated' in [8] for bisequences in $\Omega_{A}$ corresponds to 'point-separated' defined below for bisequences in $\Omega\left(L^{(n-1)}\left(G_{0}(A)\right)\right)$.) These discussions on $h_{f}$ and $\left(h_{f}\right)_{\infty}$ can straightforwardly be extended to any homomorphism $h$ of a strongly connected graph $G_{1}$ into a strongly connected graph $G_{2}$ with $r\left(G_{1}\right)=r\left(G_{2}\right)$, and $h_{\infty}$.

Let $G$ be a graph. Two bisequences $\alpha, \beta \in \Omega(G)$ are point-separated if $\mathrm{i}\left(\alpha_{i}\right) \neq \mathrm{i}\left(\beta_{i}\right)$ for all $i \in \mathbb{Z}$.

The following lemma is proved in the same way as [8, lemma 16.7].

LEMMA 6.1. Let $G_{1}$ and $G_{2}$ be graphs and let $h$ be a homomorphism of $G_{1}$ into $G_{2}$. If for each $\beta \in h_{\infty}\left(\Omega\left(G_{1}\right)\right)$, any two distinct members of $h_{\infty}^{-1}(\beta)$ are point-separated, then $h$ is mergible. 
Let $G$ be a strongly connected graph. A bisequence $\alpha \in \Omega(G)$ is positively transitive [negatively transitive] if for each positive integer $l$ and each $x \in \Pi^{(l)}(G)$, there exists $i \in \mathbb{Z}$ with $i \geq 0[i \in \mathbb{Z}$ with $i \leq-l+1]$ such that

$$
\alpha[i, i+l-1]=x \text {. }
$$

A bisequence $\alpha \in \Omega(G)$ is bilaterally transitive if $\alpha$ is both positively transitive and negatively transitive.

The result of L. R. Welch and A. M. Gleason given as theorems 11.1 and 11.2 of [8], can straightforwardly be extended to the following theorem; a similar extension in a more general setting was stated in [5].

THEOREM 6.2. Let $G_{1}$ and $G_{2}$ be strongly connected graphs with $r\left(G_{1}\right)=r\left(G_{2}\right)$, and let $h$ be a homomorphism of $G_{1}$ into $G_{2}$ with $h^{*}$ onto. Then there exists a positive integer $m(h)$ such that if $\beta \in \Omega\left(G_{2}\right)$ is bilaterally transitive, then

$$
\left|h_{\infty}^{-1}(\beta)\right|=m(h) \text {. }
$$

Furthermore, for each $\beta \in \Omega\left(G_{2}\right)$,

$$
\left|h_{\infty}^{-1}(\beta)\right| \geq m(h)
$$

and the set $h_{\infty}^{-1}(\beta)$ contains $m(h)$ members which are mutually point-separated.

Now we reach our first goal.

THEOREM 6.3. Let $G_{1}$ and $G_{2}$ be strongly connected graphs and let $h$ be a homomorphism of $G_{1}$ into $G_{2}$. Then the following statements are equivalent.

(1) $h_{\infty}$ is constant-to-one and onto.

(2) $r\left(G_{1}\right)=r\left(G_{2}\right)$ and for each $\beta \in \Omega\left(G_{2}\right)$, any two distinct members in $h_{\infty}^{-1}(\beta)$ are point-separated.

(3) $r\left(G_{1}\right)=r\left(G_{2}\right)$ and $h$ is mergible.

(4) $h^{*}$ is onto and $h$ is mergible.

(5) There is a strongly connected graph $H$, an integer $p \geq 0$, a biregular homomorphism $g$ of $H$ into $L^{(p)}\left(G_{2}\right)$, and an isomorphism $\rho:\left(\Omega\left(G_{1}\right), \sigma_{1}\right) \rightarrow(\Omega(H), \sigma)$ such that $h_{\infty}=\left(h_{G_{2}, p+1,1}\right)_{\infty} g_{\infty} \rho$.

Proof. By proposition 1.3 and theorem 6.2, (1) implies (2). By lemma 6.1, (2) implies (3). By theorem 5.9, (3) implies (5). By proposition 2.2, (5) implies (1). If $h$ is mergible, then no two distinct paths in $G_{1}$ are indistinguishable by $h$. Hence (3) and (4) are equivalent.

Thus we have a structure result for constant-to-one and onto homomorphisms of irreducible subshifts of finite type.

COROLlARY 6.4. Let $G_{1}$ and $G_{2}$ be strongly connected graphs and let $\pi:\left(\Omega\left(G_{1}\right), \sigma_{1}\right) \rightarrow\left(\Omega\left(G_{2}, \sigma_{2}\right)\right.$ be a homomorphism. Then $\pi$ is constant-to-one and onto iff there exists a strongly connected graph $H$, an integer $p \geq 0$, a biregular homomorphism $\mathrm{g}$ of $H$ into $L^{(p)}\left(G_{2}\right)$, and an isomorphism $\rho:\left(\Omega\left(G_{1}\right), \sigma_{1}\right) \rightarrow(\Omega(H), \sigma)$ such that

$$
\pi=\left(h_{G_{2}, p+1,1}\right)_{\infty} g_{\infty} \rho .
$$

Proof. This follows from theorem 6.3 and corollary 1.6 
We remark that there exists a finite procedure to determine whether (4) of theorem 6.3 holds or not for a given homomorphism between strongly connected graphs. We also remark that we can obtain completely analogous results to theorems $\mathbf{1 6 . 1}$ (a theorem of O. S. Rothaus) and 16.11 in [8] for homomorphisms of irreducible subshifts of finite type, a part of which was stated in [5] without proof.

Let $G$ be a graph and let $\alpha, \beta \in \Omega(G)$. We say that $\alpha$ and $\beta$ are totally 0 -separated if $\alpha$ and $\beta$ are point-separated. For a positive integer $p, \alpha$ and $\beta$ are totally $p$-separated if

$$
\alpha[i, i+p-1] \neq \beta[i, i+p-1] \quad \text { for all } i \in \mathbb{Z} .
$$

For other terminology see [8].

THEOREM 6.5. Let $G_{1}$ and $G_{2}$ be strongly connected graphs with $r\left(G_{1}\right)=r\left(G_{2}\right)$. Let $p$ be a positive integer. Let $f: \Pi^{(p)}\left(G_{1}\right) \rightarrow \Pi^{(1)}\left(G_{2}\right)$ be an admissible p-block map. Then the following statements are equivalent.

(1) $f_{\infty}$ is constant-to-one.

(2) $f_{\infty}$ is open and onto.

(3) $f_{\infty}$ has a cross-section.

(4) For each $\beta \in \Omega\left(G_{2}\right)$, any two distinct members of $f_{\infty}^{-1}(\beta)$ are totally $(p-1)$-separated.

Proof. Using the equivalence of (1) and (2) of theorem 6.3 and straightforwardly modifying a part of the discussions in $\S 16$ of [8] (see lemmas and theorems from 16.2 to 16.6 and their proofs together with a theorem of E. A. Michael), we first have the theorem for $p=1$. The general case can obviously be reduced to this.

COROLLARY 6.6. Let $G_{1}$ and $G_{2}$ be two strongly connected graphs with $r\left(G_{1}\right)=r\left(G_{2}\right)$ and let $\pi:\left(\Omega\left(G_{1}\right), \sigma_{1}\right) \rightarrow\left(\Omega\left(G_{2}\right), \sigma_{2}\right)$ be a homomorphism. The following statements are equivalent.

(1) $\pi$ is constant-to-one.

(2) $\pi$ is open and onto.

(3) $\pi$ has a cross-section.

(4) For each $\beta \in \Omega\left(G_{2}\right)$, any two distinct members of $\pi^{-1}(\beta)$ are separated.

Proof. This is proved using theorems 6.5 and 1.5 in the same way as [8, theorem 16.11].

Furthermore, we remark that the following generalization of [15, theorem 2] is obtained in the same way as in [15].

THEOREM 6.7. Let $G_{1}, G_{2}$, and $G_{3}$ be strongly connected graphs with $r\left(G_{1}\right)=r\left(G_{2}\right)=$ $r\left(G_{3}\right)$. Let $\pi_{1}:\left(\Omega\left(G_{1}\right), \sigma_{1}\right) \rightarrow\left(\Omega\left(G_{2}\right), \sigma_{2}\right)$ and $\pi_{2}:\left(\Omega\left(G_{2}\right), \sigma_{2}\right) \rightarrow\left(\Omega\left(G_{3}\right), \sigma_{3}\right)$ be homomorphisms. Then if $\pi_{2} \pi_{1}$ is constant-to-one, each of $\pi_{1}$ and $\pi_{2}$ is constant-to-one.

\section{Constant-to-one extensions of irreducible subshifts of finite type}

In this section, we determine, up to topological conjugacy, the subshifts of finite type which are constant-to-one extensions of a given irreducible subshift of finite type. We say that $\left(\Omega\left(G_{1}\right), \sigma_{1}\right)$ is a constant-to-one extension of $\left(\Omega\left(G_{2}\right), \sigma_{2}\right)$ if there 
exists a constant-to-one homomorphism of $\left(\Omega\left(G_{1}\right), \sigma_{1}\right)$ onto $\left(\Omega\left(G_{2}\right), \sigma_{2}\right)$. If $\left(\Omega\left(G_{1}\right), \sigma_{1}\right)$ is a constant-to-one extension of $\left(\Omega\left(G_{2}\right), \sigma_{2}\right)$ and $\left(\Omega\left(G_{2}\right), \sigma_{2}\right)$ is irreducible (i.e. $G_{2}$ is strongly connected), $\left(\Omega\left(G_{1}\right), \sigma_{1}\right)$ is not necessarily irreducible (i.e. $G_{1}$ is not necessarily strongly connected). But we do have proposition 7.1 below.

A graph $G^{\prime}=\left(P^{\prime}, A^{\prime}, \zeta^{\prime}\right)$ is a subgraph of a graph $G=(P, A, \zeta)$ if $P^{\prime} \subset P, A^{\prime} \subset A$, and $\zeta^{\prime}(a)=\zeta(a)$ for all $a \in A^{\prime}$. A maximal strongly connected subgraph of a graph $G$ is called a component of $G$.

Proposition 7.1. Let $G_{1}$ be a graph whose components are $G_{11}, \cdots, G_{1 m}$, and let $G_{2}$ be a strongly connected graph. Let there exist a constant-to-one homomorphism $\pi$ of $\left(\Omega\left(G_{1}\right), \sigma_{1}\right)$ onto $\left(\Omega\left(G_{2}\right), \sigma_{2}\right)$. Then $G_{1}$ is the union of $G_{11}, \ldots, G_{1 m}$, that is, there exists no path in $G_{1}$ going from a point of $G_{1 i}$ to a point of $G_{1 j}$ for any distinct $i, j$ with $1 \leq i, j \leq m$. Moreover, $\pi_{i}=\pi \mid \Omega\left(G_{1 i}\right)$ is constant-to-one and onto for $i=$ $1, \ldots, m$.

To prove proposition 7.1, we shall use the following lemma.

LEMMA 7.2. Let $G_{1}$ be a graph, let $G_{2}$ be a strongly connected graph and let $h$ be a homomorphism of $G_{1}$ into $G_{2}$ with $h_{\infty}$ finite-to-one and onto. Let $\beta \in \Omega\left(G_{2}\right)$ be bilaterally transitive and let $\alpha \in h_{\infty}^{-1}(\beta)$. Then $\alpha \in \Omega\left(G_{1 i}\right)$ for some component $G_{1 i}$ of $G_{1}$.

Proof. Assume that $\alpha$ is not contained in $\Omega\left(G_{1 i}\right)$ for any component $G_{1 i}$ of $G_{1}$. Then there exist components $G_{1 k}$ and $G_{1 l}$ of $G_{1}$ and $s, t \in \mathbb{Z}$ such that

$$
\begin{array}{cc}
\Pi^{(1)}\left(G_{1 k}\right) \ni \alpha_{j} & \text { for all } j<s, \\
\Pi^{(1)}\left(G_{1 l}\right) \ni \alpha_{j} & \text { for all } j>t,
\end{array}
$$

but neither $\Pi\left(G_{1 k}\right)$ nor $\Pi\left(G_{1 l}\right)$ contains $\alpha[s, t]$. Let

$$
h_{k}=h \mid \Pi^{(1)}\left(G_{1 k} \quad \text { and } \quad h_{l}=h \mid \Pi^{(1)}\left(G_{1 l}\right) .\right.
$$

Then $h_{k}$ and $h_{l}$ are homomorphisms of $G_{1 k}$ into $G_{2}$ and of $G_{1 l}$ into $G_{2}$, respectively. Since $\beta$ is negatively transitive, $\left(h_{k}\right)^{*}$ is onto, and also since $\beta$ is positively transitive, $\left(h_{l}\right)^{*}$ is onto. Since $h_{\infty}$ is finite-to-one, so are both of $\left(h_{k}\right)_{\infty}$ and $\left(h_{l}\right)_{\infty}$. Hence both of $\left(h_{k}\right)_{\infty}$ and $\left(h_{l}\right)_{\infty}$ are uniformly finite-to-one and onto.

It is known [4, p. 175]) that the inverses of a negatively [positively] transitive point (bisequence) through a finite-to-one and onto homomorphism between irreducible subshifts of finite type are also negatively [positively] transitive. Therefore since $\beta$ is negatively transitive, $\alpha$ is negatively transitive in $G_{1 k}$. (Consider a bisequence $\alpha^{\prime} \in \Omega\left(G_{1 k}\right)$ such that $\alpha_{j}^{\prime}=\alpha_{j}$ for all $j<s$ and apply the above fact to $\left(h_{k}\right)_{\infty}\left(\alpha^{\prime}\right)$.) Similarly, since $\beta$ is positively transitive, $\alpha$ is positively transitive in $G_{1 l}$.

There exists $x_{1} \in \Pi\left(G_{1 k}\right)$ such that $\bar{C}_{h_{k}}\left(h_{k}^{*}\left(x_{1}\right), t\left(x_{1}\right)\right)$ is a maximal b-compatible set for $h_{k}$. Since $\alpha$ is negatively transitive in $G_{1 k}$, there exist $s_{1}, s_{2} \in \mathbb{Z}$ with $s_{1} \leq s_{2} \leq s$ such that $\alpha\left[s_{1}, s_{2}\right]=x_{1}$. (By corollary 3.3, we may assume that the length of $x_{1}$ is greater than 0.) Also, there exists $x_{2} \in \Pi\left(G_{1 l}\right)$ such that $\mathrm{C}_{h_{1}}\left(\mathrm{i}\left(x_{2}\right), h^{*}\left(x_{2}\right)\right)$ is a maximal compatible set for $h_{l}$. Since $\alpha$ is positively transitive in $G_{1 l}$, there exist 
$t_{1}, t_{2} \in \mathbb{Z}$ with $t \leq t_{1} \leq t_{2}$ such that $\alpha\left[t_{1}, t_{2}\right]=x_{2}$. Let

$$
U=\overline{\mathrm{C}}_{h_{k}}\left(\beta\left[s_{1}, s_{2}\right], \mathrm{t}\left(\alpha_{s_{2}}\right)\right) \text { and } V=\mathrm{C}_{h_{l}}\left(\mathrm{i}\left(\alpha_{t_{1}}\right), \beta\left[t_{1}, t_{2}\right]\right) \text {. }
$$

By the above, $U$ is a maximal b-compatible set for $h_{k}$ and $V$ is a maximal compatible set for $h_{l}$. Since $\beta$ is positively transitive, there exists $t_{3} \in \mathbb{Z}$ with $t_{3}>t_{2}$ such that

$$
\mathrm{t}\left(\beta_{t_{3}}\right)=\mathrm{i}\left(\beta_{s_{1}}\right)
$$

Let $y=\beta\left[s_{1}, t_{3}\right]$. Then $\mathrm{i}(y)=\mathrm{t}(y)$. For each $j \geq 0$, let

$$
U_{j}=\overline{\mathrm{C}}_{h_{k}}\left(y^{j}, U\right) \text { and } V_{j}=\mathrm{C}_{h_{l}}\left(V, \beta\left[t_{2}+1, t_{3}\right] y^{j}\right) \text {. }
$$

Then by corollary $3.3, U_{j}$ is a maximal b-compatible set for $h_{k}$ and $V_{j}$ is a maximal compatible set for $h_{l}$. Hence, $U_{j} \neq \varnothing$ and $V_{j} \neq \varnothing$ for all $j \geq 0$. Therefore, there exists $\tilde{\alpha} \in \Omega\left(G_{1}\right)$ such that

$$
\begin{gathered}
\tilde{\alpha}\left[s_{2}+1, t_{1}-1\right]=\alpha\left[s_{2}+1, t_{1}-1\right], \\
\tilde{\alpha}\left[s_{1}-j\left(t_{3}-s_{1}+1\right), s_{2}\right] \in \overline{\mathrm{B}}_{h_{k}}\left(y^{j} \beta\left[s_{1}, s_{2}\right],\left\{\mathrm{t}\left(\alpha_{s_{2}}\right)\right\}\right) \quad \text { for all } j \geq 0,
\end{gathered}
$$

and

$$
\tilde{\alpha}\left[t_{1}, t_{3}+j\left(t_{3}-s_{1}+1\right)\right] \in \mathrm{B}_{h_{l}}\left(\left\{\mathrm{i}\left(\alpha_{t_{1}}\right)\right\}, \beta\left[t_{1}, t_{3}\right] y^{j}\right) \quad \text { for all } j \geq 0 .,
$$

Let $\tilde{\beta}=h_{\infty}(\tilde{\alpha})$. Then clearly $\tilde{\beta}$ is a periodic bisequence of period $t_{3}-s_{1}+1$ with $\tilde{\beta}\left[s_{1}, t_{3}\right]=y$. Clearly $\tilde{\alpha}$ is not periodic. But

$$
h_{\infty}\left(\sigma^{j\left(t_{3}-s_{1}+1\right)}(\tilde{\alpha})\right)=\tilde{\beta} \quad \text { for all } j \in \mathbb{Z}
$$

where $\sigma$ is the shift on $\Omega\left(G_{1}\right)$. Hence $h_{\infty}^{-1}(\tilde{\beta})$ is infinite, which is a contradiction. Thus $\alpha \in \Omega\left(G_{1 i}\right)$ for some component $G_{1 i}$ of $G_{1}$.

Proof of proposition 7.1. By corollary 1.6 , there exist positive integers $p, q, p \geq q$, and a homomorphism $h$ of $L^{(p-1)}\left(G_{1}\right)$ into $G_{2}$ such that

$$
\pi=h_{\infty}\left(h_{G_{1}, p, q}\right)_{\infty}^{-1} \text {. }
$$

Clearly $h_{\infty}$ is constant-to-one and onto. Put $H=L^{(p-1)}\left(G_{1}\right)$ and put $H_{i}=L^{(p-1)}\left(G_{1 i}\right)$ for $i=1, \ldots, m$. Then it is easy to see that $H_{1}, \ldots, H_{m}$ are all the components of $H$. Let

$$
h_{i}=h \mid \Pi^{(1)}\left(H_{i}\right) \quad \text { for } i=1, \ldots, m \text {. }
$$

Then clearly, $h_{i}$ is a homomorphism of $H_{i}$ into $G_{2}$ with $\left(h_{i}\right)_{\infty}$ finite-to-one.

Let $\beta$ be a bilaterally transitive bisequence in $\Omega\left(G_{2}\right)$. Then, by lemma 7.2, each $\alpha \in \Omega(H)$ such that $h_{\infty}(\alpha)=\beta$, is contained in some $\Omega\left(H_{i}\right), 1 \leq i \leq m$. Let

$$
\left\{i_{1}, \ldots, i_{l}\right\}=\left\{i \mid 1 \leq i \leq m, \quad \Omega\left(H_{i}\right) \cap h_{\infty}^{-1}(\beta) \neq \varnothing\right\} .
$$

Assume that there exists $\alpha^{\prime} \in \Omega(H)$ such that $\alpha^{\prime} \not \Omega\left(H_{i_{1}}\right) \cup \cdots \cup \Omega\left(H_{i_{l}}\right)$. Let $\gamma=h_{\infty}\left(\alpha^{\prime}\right)$. Since $\beta$ is bilaterally transitive, $h_{i_{j}}^{*}$ is onto and so is $\left(h_{i_{j}}\right)_{\infty}$ for $j=1, \cdots, l$. Hence $\left(h_{i_{j}}\right)_{\infty}$ is finite-to-one and onto for $j=1, \ldots, l$. By theorem 6.2,

for $j=1, \ldots, l$. Hence

$$
\left|\left(h_{i_{j}}\right)_{\infty}^{-1}(\beta)\right| \leq\left|\left(h_{i_{j}}\right)_{\infty}^{-1}(\gamma)\right|
$$

$$
\left|h_{\infty}^{-1}(\beta)\right|=\sum_{j=1}^{l}\left|\left(h_{i_{j}}\right)_{\infty}^{-1}(\beta)\right|<1+\sum_{j=1}^{l}\left|\left(h_{i_{j}}\right)_{\infty}^{-1}(\gamma)\right| \leq\left|h_{\infty}^{-1}(\gamma)\right|,
$$

which is a contradiction because $h_{\infty}$ is constant-to-one and onto. Therefore $\Omega\left(H_{i_{1}}\right) \cup \cdots \cup \Omega\left(H_{i_{l}}\right)=\Omega(H)$. This implies that $\left\{i_{1}, \ldots, i_{l}\right\}=\{1, \ldots, m\}$, 
$\Omega(H)=\Omega\left(H_{1}\right) \cup \cdots \cup \Omega\left(H_{m}\right)$ and $\left(h_{i}\right)_{\infty}$ is finite-to-one and onto for $i=1, \ldots, m$. Hence for each $\beta^{\prime} \in \Omega\left(G_{2}\right)$,

$$
\left|h_{\infty}^{-1}\left(\beta^{\prime}\right)\right|=\sum_{i=1}^{m}\left|\left(h_{i}\right)_{\infty}^{-1}\left(\beta^{\prime}\right)\right|,
$$

and by theorem 6.2 ,

$$
\left|\left(h_{i}\right)_{\infty}^{-1}(\beta)\right| \leq\left|\left(h_{i}\right)_{\infty}^{-1}\left(\beta^{\prime}\right)\right|,
$$

$i=1, \ldots, m$. Therefore, since $h_{\infty}$ is constant-to-one, each $\left(h_{i}\right)_{\infty}$ must be constant-toone.

Since $\Omega(H)=\Omega\left(H_{1}\right) \cup \cdots \cup \Omega\left(H_{m}\right), \Omega\left(G_{1}\right)=\Omega\left(G_{11}\right) \cup \cdots \cup \Omega\left(G_{1 m}\right)$. Hence there is no path in $G_{1}$ going from a point of $G_{1 i}$ to a point of $G_{1 j}$ for any distinct $i, j$ with $1 \leq i, j \leq m$. Since

$$
\pi_{i}=\left(h_{i}\right)_{\infty}\left(h_{G_{1}, p, q}\right)_{\infty}^{-1},
$$

$\pi_{i}$ is constant-to-one and onto.

The following is the main theorem of this paper. In view of proposition 2.3 , it determines constructively, up to topolological conjugacy, the subshifts of finite type which are constant-to-one extensions of a given irreducible subshift of finite type.

THEOREM 7.3. Let $G_{1}$ be a graph and $G_{2}$ a strongly connected graph. Then $\left(\Omega\left(G_{1}\right), \sigma_{1}\right)$ is a constant-to-one extension of $\left(\Omega\left(G_{2}\right), \sigma_{2}\right)$ iff there is a biregular extension $H$ of $L^{(p)}\left(G_{2}\right)$ for some integer $p \geq 0$ such that $\left(\Omega\left(G_{1}\right), \sigma_{1}\right)$ is topologically conjugate to $(\Omega(H), \sigma)$.

Proof. Assume that there is a constant-to-one homomorphism $\pi$ of $\left(\Omega\left(G_{1}\right), \sigma_{1}\right)$ onto $\left(\Omega\left(G_{2}\right), \sigma_{2}\right)$. Then from proposition 7.1, $G_{1}$ is the union of its components $G_{11}, \ldots, G_{1 m}$ and $\pi \mid \Omega\left(G_{1 i}\right)$ is constant-to-one and onto for $i=1, \ldots, m$. Put

$$
\pi_{i}=\pi \mid \Omega\left(G_{1 i}\right) \quad \text { for } i=1, \ldots, m .
$$

It follows from corollary 6.4 that for each $i=1, \ldots, m$, there exists a strongly connected graph $H_{i}$, an integer $p_{i} \geq 0$, and a biregular homomorphism $g_{i}$ of $H_{i}$ into $L^{\left(p_{i}\right)}\left(G_{2}\right)$ such that $\left(\Omega\left(H_{i}\right), \sigma_{i}^{\prime}\right)$ and $\left(\Omega\left(G_{1 i}\right), \sigma_{1 i}\right)$ are topologically conjugate. Let $p=\max \left\{p_{1}, \ldots, p_{m}\right\}$. Consider the homomorphism $g_{i}^{\left(p-p_{i}\right)}$ of $L^{\left(p-p_{i}\right)}\left(H_{i}\right)$ into $L^{\left(p-p_{i}\right)}\left(L^{\left(p_{i}\right)}\left(G_{2}\right)\right)$, (see the paragraph before lemma 5.6). It is easy to see that $g_{i}^{\left(p-p_{i}\right)}$ is biregular. Therefore, since $L^{(p)}\left(G_{2}\right)$ is isomorphic to $L^{\left(p-p_{i}\right)}\left(L^{\left(p_{i}\right)}\left(G_{2}\right)\right), L^{\left(p-p_{i}\right)}\left(H_{i}\right)$ is a biregular extension of $L^{(p)}\left(G_{2}\right)$. It follows that $\left(\Omega\left(L^{\left(p-p_{i}\right)}\left(H_{i}\right)\right), \sigma_{i}^{\prime \prime}\right)$ is topologically conjugate to $\left(\Omega\left(G_{1 i}\right), \sigma_{1 i}\right)$ for $i=1, \ldots, m$. Let $H$ be the union of the graphs $L^{\left(p-p_{i}\right)}\left(H_{i}\right), i=1, \ldots, m$. Then, by the above, $(\Omega(H), \sigma)$ is topologically conjugate to $\left(\Omega\left(G_{1}\right), \sigma_{1}\right)$. Moreover, since $H$ is the union of its components each of which is a biregular extension of $L^{(p)}\left(G_{2}\right), H$ is a biregular extension of $L^{(p)}\left(G_{2}\right)$.

The converse is clear from proposition 2.2 .

There is a remarkable spectral property of matrices concerning topological conjugacy of subshifts of finite type. It follows directly from the well-known theorem of Williams, [21], (characterizing topological conjugacy of subshifts of finite type by 
'strong shift equivalence' of matrices defining the subshifts), and a result of Flanders (see $\left[9\right.$, p. 106]) that if two subshifts of finite type $\left(\Omega\left(G_{1}\right), \sigma_{1}\right)$ and $\left(\Omega\left(G_{2}, \sigma_{2}\right)\right.$ are topologically conjugate, then the elementary divisors not divisible by $\lambda$ of $M\left(G_{1}\right)$ and those of $M\left(G_{2}\right)$ are the same, where $\lambda$ is the indeterminate. (See also [19].) But the converse of this does not hold by example 3 of [21].

As stated in $\S 1$, there are also remarkable spectral properties of matrices concerning finite-to-one extensions of subshifts of finite type. In [17], the author showed that for two subshifts of finite type $\left(\Omega\left(G_{1}\right), \sigma_{1}\right)$ and $\left(\Omega\left(G_{2}\right), \sigma_{2}\right)$, if there is a finite-to-one homomorphism of $\left(\Omega\left(G_{1}\right), \sigma_{1}\right)$ onto $\left(\Omega\left(G_{2}\right), \sigma_{2}\right)$, then the characteristic polynomial of $M\left(G_{2}\right)$ divides the characteristic polynomial of $M\left(G_{1}\right)$, mod powers of $\lambda$. Furthermore, Kitchens, [10], showed that if $\left(\Omega\left(G_{1}\right), \sigma_{1}\right)$ and $\left(\Omega\left(G_{2}\right), \sigma_{2}\right)$ are irredicible subshifts of finite type and there is a finite-to-one homomorphism of $\left(\Omega\left(G_{1}\right), \sigma_{1}\right)$ onto $\left(\Omega\left(G_{2}\right), \sigma_{2}\right)$, then the block of the Jordan form of $M\left(G_{2}\right)$ with non-zero eigenvalues is a principal submatrix of the Jordan form of $M\left(G_{1}\right)$. In [10], Kitchens also showed that the converse does not hold. The following theorem is a result along the above lines.

THEOREM 7.4. Let $G_{1}$ and $G_{2}$ be strongly connected graphs. If there exists a constantto-one homomorphism of $\left(\Omega\left(G_{1}\right), \sigma_{1}\right)$ onto $\left(\Omega\left(G_{2}\right), \sigma_{2}\right)$, then the elementary divisors not divisible by $\lambda$ of $M\left(G_{2}\right)$ is contained in the elementary divisors of $M\left(G_{1}\right)$.

Proof. The result follows from theorem 7.3 , proposition 2.5 , the spectral property of matrices concerning topological conjugacy stated above, and the fact that $\left(\Omega\left(G_{2}\right), \sigma_{2}\right)$ and $\left(\Omega\left(L^{(p)}\left(G_{2}\right)\right), \sigma_{2}^{\prime}\right)$ are topologically conjugate.

Marcus [13] proved that for any strongly connected graph $G$ with $r(G)=n$, where $n$ is a positive integer, there is a strongly connected graph $G^{\prime}$ such that each row sum of $M\left(G^{\prime}\right)$ is $n$, each column sum of $M\left(G^{\prime}\right)$ is $n$, and $\left(\Omega\left(G^{\prime}\right), \sigma^{\prime}\right)$ is topologically conjugate to $(\Omega(G), \sigma)$. It is easy to see that $G^{\prime}$ is a biregular extension of $G_{0}(A)$ where $A$ is the set of $n$ symbols. (See [16, lemma 1]. As for $G_{0}(A)$, see $\S 1$.) Therefore, by proposition 2.2 , every irreducible subshift of finite type $(\Omega(G), \sigma)$ with $r(G)=n$ is a constant-to-one extension of the full shift system on $n$ symbols. Since $r(G)=n$ for every subshift of finite type $(\Omega(G), \sigma)$ which is a finite-to-one extension of the full shift system on $n$ symbols, we conclude that every irreducible subshift of finite type which is a finite-to-one extension of a full shift system, is also a constant-to-one extension of the full shift system. The question arises of whether every irreducible subshift of finite type which is a finite-to-one extension of an irreducible subshift of finite type, is also a constant-to-one extension of the irreducible subshift of finite type. The following example shows that the answer is negative.

Let $G_{1}$ and $G_{2}$ be graphs with

$$
M\left(G_{1}\right)=\left(\begin{array}{cccc}
0 & 1 & 0 & 1 \\
1 & 0 & 0 & 1 \\
0 & 0 & 1 & 1 \\
1 & 0 & 1 & 0
\end{array}\right) \text { and } M\left(G_{2}\right)=\left(\begin{array}{ll}
1 & 1 \\
2 & 0
\end{array}\right)
$$


Clearly $G_{1}$ and $G_{2}$ are strongly connected. Since $M\left(G_{1}\right) R=R M\left(G_{2}\right)$ where

$$
R=\left(\begin{array}{ll}
1 & 0 \\
1 & 0 \\
1 & 0 \\
0 & 1
\end{array}\right)
$$

it follows from proposition 2.4 that there is a regular homomorphism of $G_{1}$ into $G_{2}$. Hence $\left(\Omega\left(G_{1}\right), \sigma_{1}\right)$ is a finite-to-one extension of $\left(\Omega\left(G_{2}\right), \sigma_{2}\right)$. But the elementary divisors of $M\left(G_{1}\right)$ are $\lambda-2, \lambda-1$, and $(\lambda+1)^{2}$, whereas the elementary divisors of $M\left(G_{2}\right)$ are $\lambda-2$ and $\lambda+1$. Therefore, by theorem 7.4, $\left(\Omega\left(G_{1}\right), \sigma_{1}\right)$ is not a constant-to-one extension of $\left(\Omega\left(G_{2}\right), \sigma_{2}\right)$. (Note also that both $\left(\Omega\left(G_{1}\right), \sigma_{1}\right)$ and $\left(\Omega\left(G_{2}\right), \sigma_{2}\right)$ are constant-to-one extensions of the full shift system on 2 symbols.)

The converse of theorem 7.4 does not hold. An example of Kitchens [10] shows that if $G_{1}$ and $G_{2}$ are strongly connected graphs such that the elementary divisors of $M\left(G_{1}\right)$ contains the elementary divisors of $M\left(G_{2}\right),\left(\Omega\left(G_{1}\right), \sigma_{1}\right)$ is not necessarily even an extension of $\left(\Omega\left(G_{2}\right), \sigma_{2}\right)$.

Question. Does the converse of theorem 7.4 hold under the condition that $\left(\Omega\left(G_{1}\right), \sigma_{1}\right)$ is a finite-to-one extension of $\left(\Omega\left(G_{2}\right), \sigma_{2}\right)$ ?

\section{Concluding remarks}

Finally, to show that there are other applications of induced regular homomorphisms and induced b-regular homomorphisms, we state some results omitting proofs.

Let $G_{1}$ and $G_{2}$ be strongly connected graphs with $r\left(G_{1}\right)=r\left(G_{2}\right)$ and let $h$ be a homomorphism of $G_{1}$ into $G_{2}$ with $h^{*}$ onto. Let $m(h)$ be as in theorem 6.2 . One can prove that

$$
m(\tilde{h})=m(\overline{\tilde{h}})=m(h)
$$

and if $v$ is any point of $G_{2}, m(h)$ equals the maximum number of mutually disjoint maximal b-compatible [compatible] sets for $\tilde{h}$ [for $\tilde{\tilde{h}}$ ] contained in $\phi_{\tilde{h}}^{-1}(v)$ [in $\phi_{\hat{h}}^{\bar{h}^{1}}(v)$ ]. One can also prove that $m(h)=1$ iff $U \cap V \neq \varnothing(|U \cap V|=1)$ for any maximal compatible set $U$ for $h$ and any maximal b-compatible set $V$ for $h$ with $\phi_{h}(U)=$ $\phi_{h}(V)$.

Let $h$ be a regular [b-regular] homomorphism of a graph $G_{1}$ into a graph $G_{2}$. Let $p$ be a non-negative integer. Then $h$ is said to be $p$ definite if for any $x_{1}$, $x_{2} \in \Pi^{(p)}\left(G_{1}\right), h^{*}\left(x_{1}\right)=h^{*}\left(x_{2}\right)$ implies $\mathrm{t}\left(x_{1}\right)=\mathrm{t}\left(x_{2}\right)\left[\mathrm{i}\left(x_{1}\right)=\mathrm{i}\left(x_{2}\right)\right]$, and $h$ is said to be definite if $h$ is $p$ definite for some non-negative integer $p$.

A definite regular homomorphism is considered to be a generalization of the state transition diagram of a finite automaton having a definite table, which was introduced in [20]. The properties of definite tables and a practical decision procedure for definiteness of tables presented in [20], can straightforwardly be extended to definite regular [b-regular] homomorphisms of graphs.

Let $G_{1}$ and $G_{2}$ be strongly connected graphs with $r\left(G_{1}\right)=r\left(G_{2}\right)$, and let $h$ be a homomorphism of $G_{1}$ into $G_{2}$ with $h^{*}$ onto. Then one can show that the induced 
regular[b-regular] homomorphism $\tilde{h}[\overline{\bar{h}}]$ of $h$ is $p$ definite iff $h$ is $p$ b-bundle-mergible [ $p$ bundle-mergible] and $m(h)=1$. (Cf. [14, theorem 5].) Therefore a criterion for bijectivity of $h_{\infty}$ can be obtained, that is, $h_{\infty}$ is one-to-one and onto iff both $\tilde{h}$ and $\overline{\tilde{h}}$ are definite.

\section{REFERENCES}

[1] R. L. Adler \& B. Marcus. Topological entropy and equivalence of dynamical systems. Memoirs Amer. Math. Soc. 219 (1979).

[2] C. Berge. Graphs and Hypergraphs. North-Holland: Amsterdam, 1973.

[3] E. M. Coven \& M. E. Paul. Endomorphisms of irreducible subshifts of finite type. Math. Systems Theory 8 (1974), 167-175.

[4] E. M. Coven \& M. E. Paul. Sofic systems. Israel J. Math. 20 (1975), 165-177.

[5] E. M. Coven \& M. E. Paul. Finite procedures for sofic systems. Monatsh. Math. 83 (1977), 265-278.

[6] F. R. Gantmacher. The Theory of Matrices, Vols. I and II. Chelsea: New York, 1959.

[7] F. Harary. Graph Theory. Addison-Wesley: Reading, Mass., 1969.

[8] G. A. Hedlund. Endomorphisms and automorphisms of the shift dynamical system. Math. Systems Theory 3 (1969), 320-375.

[9] N. Jacobson. Lectures in Abstract Algebra, Vol. II. Springer-Verlag: New York, 1953.

[10] B. Kitchens. An invariant for continuous factors of Markov shifts. Proc. Amer. Math. Soc. 83 $\{1981\}, 825-828$.

[11] B. Kitchens. Continuity properties of factor maps in ergodic theory. Ph.D. Thesis, University of North Carolina, Chapel Hill (1981).

[12] B. G. Klein. Homomorphisms of symbolic dynamical systems. Math. Systems Theory 6 (1972), $107-122$.

[13] B. Marcus. Factors and extensions of full shifts. Monatsh. Math. 88 (1979), 239-247.

[14] M. Nasu. Local maps inducing surjective global maps of one-dimensional tessellation automata. Math. Systems Theory 11 (1978), 327-351.

[15] M. Nasu. Indecomposable local maps of tessellation automata. Math. Systems Theory 13 (1979), 81-93.

[16] M. Nasu. An interconnection of local maps inducing onto global maps. Discrete Applied Math. 2 (1980), 125-150.

[17] M. Nasu. Uniformly finite-to-one and onto extensions of homomorphisms between strongly connected graphs. Discrete Math. 39 (1982), 171-197.

[18] W. Parry. A finitary classification of topological Markov chains and sofic systems. Bull. London Math. Soc. 9 (1977), 86-92.

[19] W. Parry \& R. F. Williams. Block coding and a zeta function for finite Markov chains. Proc. London Math. Soc. (3) 35 (1977), 483-495.

[20] M. Perles, M. O. Rabin, \& E. Shamir. The theory of definite automata. IEEE Trans. Electr. Comp. EC-12 (1963), 233-243.

[21] R. F. Williams. Classification of subshifts of finite type. Ann. of Math. 98 (1973), 120-153; Errata: Ann. of Math. 99 (1974), 380-381. 